Delft University of Technology

\title{
Aerodynamic investigation of an over-the-wing propeller for distributed propulsion
}

Marcus, E. A.P.; De Vries, R.; Kulkarni, A. Raju; Veldhuis, L. L.M.

DOI

10.2514/6.2018-2053

Publication date

2018

Document Version

Final published version

Published in

AIAA Aerospace Sciences Meeting

\section{Citation (APA)}

Marcus, E. A. P., De Vries, R., Kulkarni, A. R., \& Veldhuis, L. L. M. (2018). Aerodynamic investigation of an over-the-wing propeller for distributed propulsion. In AIAA Aerospace Sciences Meeting (210059 ed.). [AIAA 2018-2053] American Institute of Aeronautics and Astronautics Inc. (AIAA). https://doi.org/10.2514/6.20182053

\section{Important note}

To cite this publication, please use the final published version (if applicable).

Please check the document version above.

\section{Copyright}

Other than for strictly personal use, it is not permitted to download, forward or distribute the text or part of it, without the consent of the author(s) and/or copyright holder(s), unless the work is under an open content license such as Creative Commons.

Takedown policy

Please contact us and provide details if you believe this document breaches copyrights.

We will remove access to the work immediately and investigate your claim. 
Green Open Access added to TU Delft Institutional Repository

'You share, we take care!' - Taverne project

https://www.openaccess.nl/en/you-share-we-take-care

Otherwise as indicated in the copyright section: the publisher is the copyright holder of this work and the author uses the Dutch legislation to make this work public. 


\title{
Aerodynamic Investigation of an Over-the-Wing Propeller for Distributed Propulsion
}

\author{
E.A.P. Marcus*, R. de Vries ${ }^{\dagger}$, A. Raju Kulkarni ${ }^{\ddagger}$ L.L.M. Veldhuis ${ }^{\S}$ \\ Delft University of Technology, Delft, 2629 HS, The Netherlands
}

\begin{abstract}
This paper addresses the aerodynamic performance and numerical modeling of overthe-wing propellers. Installing the propeller above a wing has the potential to increase wing lift-to-drag ratio, high-lift capabilities, and to reduce flyover noise. However, the prediction of its performance is difficult, since research on the aerodynamic interaction effects of over-the-wing propellers has been limited so far. For this reason, an exploratory wind tunnel campaign was performed with a wing featuring a fowler flap. A single propeller was installed above the wing at different chordwise locations and inclination angles. Wing surface-pressure and wake-pressure measurements showed strong, bilateral aerodynamic coupling between the propeller and wing. A configuration with the propeller attached to the flap showed wing lift increases of $8 \%$ and $3 \%$ in cruise and high-lift conditions, respectively. The key findings of the wind tunnel campaign were used to validate a low-fidelity numerical tool, which combines a non-uniform inflow blade-element model for the propeller, a panel method for the wing, and a vortex lattice model for the propeller slipstream. The numerical model was used to assess the effect of propeller axial location and diameter. Results indicated that the optimal axial propeller position is near the trailing edge of the wing, and that reducing the propeller diameter at constant thrust coefficient at this location is beneficial for distributed propulsion applications. The tool allows a rapid computation of over-the-wing propeller and wing performance in cruise conditions. This enables an efficient design space exploration during the conceptual design process of such configurations.
\end{abstract}

\section{Nomenclature}

Latin Symbols

$\begin{array}{llll}a & \text { Axial acceleration factor [-] } & n & \text { Propeller rotational speed [Hz] } \\ b & \text { Tangential acceleration factor [-] } & p & \text { Static pressure [Pa] } \\ c & \text { Wing chord length }[\mathrm{m}] & p_{\mathrm{t}} & \text { Total pressure [Pa] } \\ C_{D_{p}} & \text { Wing pressure drag coefficient, } \frac{D_{p}}{\frac{1}{2} \rho_{\infty} V_{\infty}^{2} S_{\mathrm{ref}}}[-] & r & \text { Propeller radial coordinate [m] } \\ C_{L} & \text { Wing lift coefficient, } \frac{L}{\frac{1}{2} \rho_{\infty} V_{\infty}^{2} S_{\mathrm{ref}}}[-] & s & \text { Slipstream acceleration factor [-] } \\ C_{p} & \text { Pressure coefficient, } \frac{p-p_{\infty}}{\frac{1}{2} \rho_{\infty} V_{\infty}^{2}}[-] & S_{\mathrm{ref}} & \text { Wing reference area, } c \cdot D_{\mathrm{P}}\left[\mathrm{m}^{2}\right] \\ C_{p_{\mathrm{t}}} & \text { Total pressure coefficient, } \frac{p_{\mathrm{t}}-p_{\infty}}{\frac{1}{2} \rho_{\infty} V_{\infty}^{2}}[-] & t & \text { Time [s] } \\ C_{T} & \text { Thrust coefficient, } \frac{T}{\rho_{\infty} n^{2} D_{\mathrm{P}}^{4}}[-] & T & \text { Propeller thrust }[\mathrm{N}] \\ D_{p} & \text { Wing pressure drag }[\mathrm{N}] & T_{C}^{*} & \text { Thrust coefficient, } \frac{T}{\frac{1}{2} \rho_{\infty} V_{\infty}^{2} S_{\mathrm{ref}}}[-] \\ D_{\mathrm{P}} & \text { Propeller diameter }[\mathrm{m}] & u & \text { Axial induced velocity }[\mathrm{m} / \mathrm{s}] \\ J & \text { Advance ratio, } \frac{V_{\infty}}{n D_{\mathrm{p}}}[-] & V & \text { Velocity magnitude }[\mathrm{m} / \mathrm{s}] \\ L & \text { Wing lift [N] } & V_{\mathrm{a}} & \text { Axial blade element velocity }[\mathrm{m} / \mathrm{s}]\end{array}$

*MSc. Student, Flight Performance and Propulsion (FPP) Section, Faculty of Aerospace Eng., Pepijn.Marcus@gmail.com.

$\dagger$ PhD. Candidate, FPP Section, Faculty of Aerospace Engineering, R.deVries@tudelft.nl, AIAA member.

$\ddagger$ PhD. Candidate, FPP Section, Faculty of Aerospace Engineering, AIAA member.

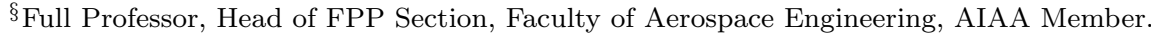




\section{Greek Symbols}

$\begin{array}{ll}\Delta(\quad) \quad \text { Increase w.r.t. propeller-off conditions } \\ & \text { or increase w.r.t. previous time step [-] } \\ \alpha & \text { Angle of attack [deg] } \\ \Gamma & \text { Circulation }\left[\mathrm{m}^{2} / \mathrm{s}\right] \\ \delta & \text { Flap deflection angle }[\mathrm{deg}] \\ \eta & \text { Propulsive efficiency [-] } \\ \theta & \text { Propeller azimuthal coordinate }[\mathrm{rad}] \\ \rho & \text { Density }\left[\mathrm{kg} / \mathrm{m}^{3}\right] \\ \Omega & \text { Propeller rotational speed }[\mathrm{rad} / \mathrm{s}]\end{array}$

\section{Additional sub- and superscripts}

$\begin{array}{ll} & \text { Propeller reference frame } \\ 0 & \text { Propeller disk location } \\ \infty & \text { Freestream conditions } \\ \text { iso } & \text { Isolated } \\ \text { P } & \text { Propeller } \\ \text { prop. on } & \text { Propeller-on conditions } \\ \text { prop. off } & \text { Propeller-off conditions } \\ \text { Ss } & \text { Slipstream } \\ \text { tr } & \text { boundary-layer transition } \\ \text { w } & \text { Wing }\end{array}$

\section{Introduction}

Emissions, noise, fuel burn, and field length are expected to be the main challenges for future aviation. ${ }^{1}$ In order to meet the stringent targets set by the National Aeronautics and Space Administration (NASA) ${ }^{2}$ and the European Commission (EC), ${ }^{3}$ radical changes in aircraft design are required. This has led to an increased interest in Hybrid-Electric Propulsion (HEP) in the recent years. ${ }^{4-8}$ HEP presents possible benefits in terms of energy conversion and transmission efficiency. Furthermore, it allows a more aerodynamically efficient integration of propulsors into the airframe. ${ }^{4}$

A possible synergistic solution is the combination of HEP and distributed over-the-wing (OTW) propellers. The use of distributed propulsion is partially enabled by the hybrid-electric powertrain, which not only leads to an increase in effective bypass ratio, ${ }^{7}$ but also allows placing propellers of reduced diameter above the wing without large pylons or a significant offset between the thrust line and the center of gravity of the aircraft. The placement of propellers above a wing entails several benefits when compared to conventional tractor configurations. Firstly, it eliminates installation challenges in terms of ground clearance. Secondly, it can increase wing lift-to-drag ratio in both cruise ${ }^{9}$ and high-lift ${ }^{10}$ conditions. Thirdly, the array of propellers can be attached to the flap mechanism, providing a degree of thrust vectoring in low-speed conditions ${ }^{\mathrm{a}}$. Finally, due to the shielding effects of the wing, flyover noise can be reduced. ${ }^{11,12}$

However, in the proposed configuration the propeller is subjected to increased inflow velocities and a vertical velocity gradient. This is detrimental for propeller efficiency ${ }^{10,13}$ and leads to unsteady blade loading, thus increasing vibrations and noise production. ${ }^{14}$ Moreover, interaction between the propeller tip vortices and the boundary layer may cause flow separation. ${ }^{15}$ It is therefore evident that the aerodynamic performance of the propeller and wing cannot be studied separately.

In the case of distributed-propulsion systems, these interaction effects have a large impact on wing sizing, ${ }^{16}$ and thus they should be included in the first stages of the design process. Although there are low-fidelity methods which estimate these effects for tractor-propeller configurations ${ }^{17}$ no suitable models exist for OTW configurations. This can be attributed to the limited application of OTW systems up till today on one hand, and to the difficulty of modeling such systems on the other. While tractor propellers affect the dynamic pressure and angle of attack perceived by the wing, ${ }^{13}$ OTW propellers affect the upper-surface pressure distribution, ${ }^{18}$ hence changing the effective airfoil shape. Modeling this effect requires a detailed understanding of the aerodynamic interaction effects. However, existing studies on over-the-wing propellers ${ }^{9,10,13,15,18,19}$ are limited, and on occasions contradictory. While some authors have found moderate ${ }^{10}$ to drastic ${ }^{13}$ reductions in propulsive efficiency, others have found an increase in propulsive efficiency. ${ }^{9}$ Analogously, most authors conclude that OTW propellers reduce the drag of the wing, ${ }^{9,13}$ but for some configurations it has been found to increase. ${ }^{18}$ Thus, it is difficult to extrapolate the findings of these studies to distributed-propulsion configurations, where the ratio between the propeller diameter and wing chord is smaller than for conventional twin- or four-engine aircraft. Furthermore, previous inquiries give no insight as to how the over-the-wing propeller can improve high-lift capabilities through thrust vectoring.

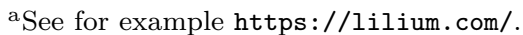


For these reasons, the purpose of this study is to develop a method capable of estimating the aerodynamic performance of OTW systems. To this end, a two-step approach is adopted. First, an exploratory wind-tunnel experiment is carried out to clarify the aforementioned contradictions, and to assess the impact of smaller OTW propellers in both cruise and high-lift conditions. The key findings are formalized in the second part of the study, which consists of the development of a numerical model. The model is capable of estimating propeller and wing performance with low computational cost. Since the dominant aerodynamic phenomena are not readily understood at this stage, the investigation will be limited to a single propeller in an over-the-wing configuration. In this way, the mutual effects between propeller and wing can be identified without the interaction between multiple propellers.

This paper presents the experimental setup of the OTW configuration in Section II. Afterwards, Section III discusses the methodology of the numerical model, the validation of its individual components, and its limitations. Subsequently, results of the experimental investigation are presented in Section IV and compared to numerical simulations, to assess the accuracy and applicability of the complete model. Furthermore, the numerical tool is used to perform an analysis of the sensitivity of OTW configurations to propeller axial position and diameter.

\section{Experimental Setup}

The wind tunnel (WT) campaign was carried out in low-turbulence tunnel at the Delft University of Technology, which has a cross-section of $1.8 \mathrm{~m}$ by $1.25 \mathrm{~m}$. This wind tunnel has a maximum velocity of 120 $\mathrm{m} / \mathrm{s}$ and freestream turbulence limited to a level of $0.02 \%$ for velocities below $40 \mathrm{~m} / \mathrm{s}^{20}$

\section{II.A. Model Description}

In order to simulate an OTW propeller configuration, a propeller was positioned on the suction side of a wing mounted vertically in the wind-tunnel test section, as depicted in Figures 1 and 2. The wing spanned the full height of the test section and was placed on a turntable, which could be rotated to change the angle of attack. It featured a chord of $0.6 \mathrm{~m}$ and an NLF-MOD22B airfoil designed at Delft University of Technology for low speed applications. ${ }^{21}$ The airfoil presents a maximum thickness-to-chord ratio of 0.17 at $35 \%$ chord and a fowler flap of $30 \%$ chord length. The main dimensions are indicated in Figure 1.
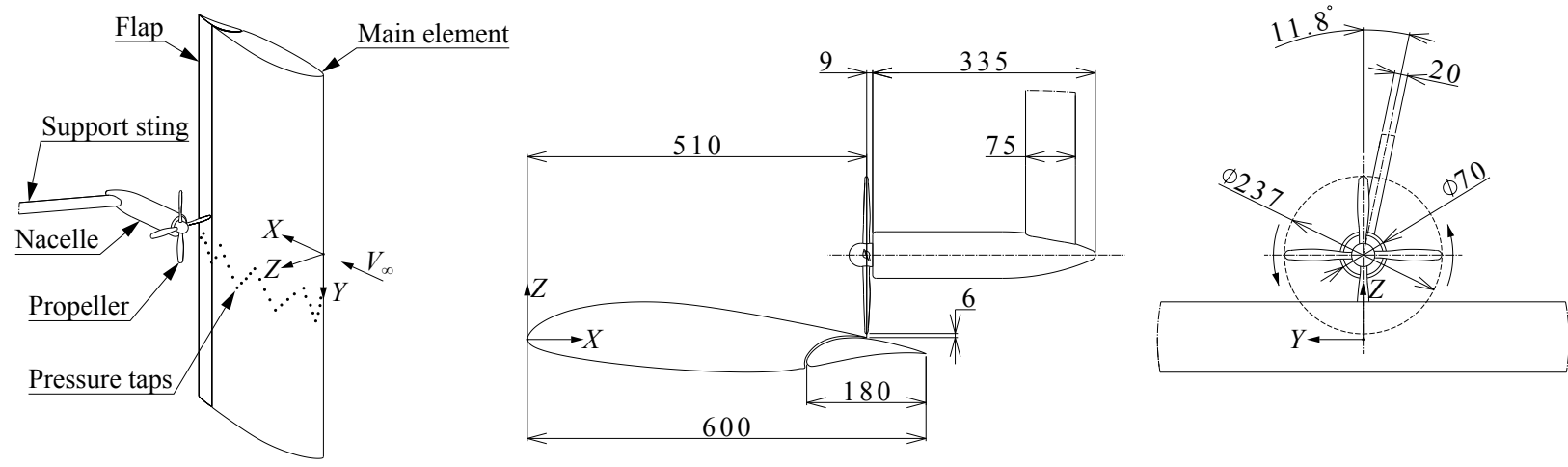

Figure 1. Isometric (left), top (middle) and front (right) views of the wind tunnel setup, including component designation, coordinate system and direction of rotation of the propeller. All dimensions are in $\mathrm{mm}$.

The four-bladed propeller model has a diameter of $D_{\mathrm{P}}=0.237 \mathrm{~m}\left(D_{\mathrm{P}} / c=0.395\right)$, a blade chord of $7.8 \%$ of the propeller diameter, and a pitch angle of $23^{\circ}$ at $75 \%$ of the propeller radius. More detailed information regarding the propeller geometry can be found in the work of Sinnige et al. ${ }^{22}$ The propeller was driven by a $7.5 \mathrm{hp}$ three-phase induction motor housed in a nacelle of $0.07 \mathrm{~m}$ diameter, positioned by means of a support sting which could be traversed along all three axes. The support sting was installed under a small inclination angle as depicted in Figure 1, in order to position the propeller axis at the spanwise location of the pressure ports (see Section II.B) when the traverse mechanism was set at its neutral position along the Y-axis.

\section{II.B. Measurement Techniques}

In the experiment two main variables were measured: the pressure distribution on the wing surface and the total pressure distribution in a wake plane (a YZ-plane downstream of the model). The former was measured 

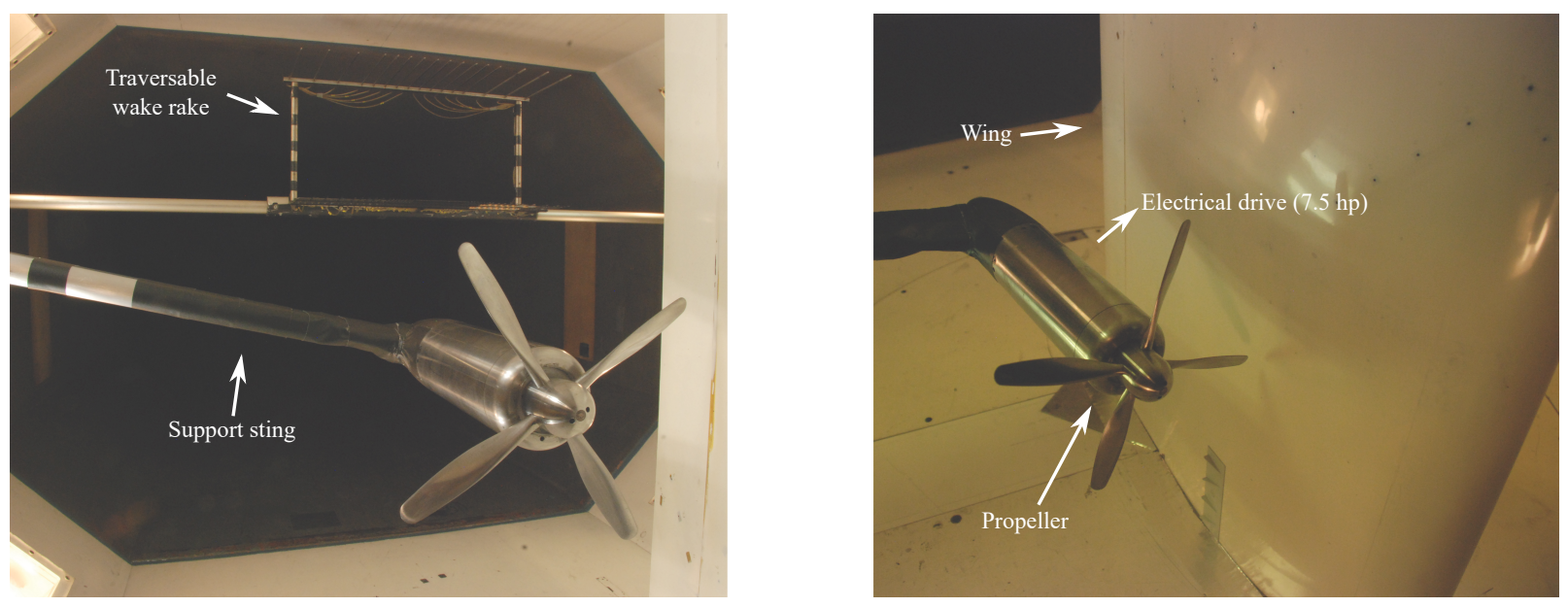

Figure 2. Photos of the wind tunnel setup, with propeller, wing, propeller support sting and wake rake indicated.

in order to quantify the effect of the propeller on the wing's pressure distribution, lift, and pressure drag, while the latter provided qualitative information regarding the time-average loading on the propeller disk and the displacement of the slipstream and wing wake. Additionally, an Optris PI640 infrared (IR) camera was installed during all measurements to observe the location of boundary-layer transition.

In order to obtain the surface pressure distribution, the wing model featured 54 static pressure ports on the main element and 27 on the flap, distributed over the pressure and suction sides. The ports were located along a zigzag path extending over a spanwise interval of $100 \mathrm{~mm}$, as indicated in Figure 1. For each configuration, the propeller was traversed in spanwise direction to resolve the wing pressure distribution, covering a span of $1.5 D_{\mathrm{P}}$.

Wake plane pressure measurements were performed with a horizontal wake rake located 1.25 chord-lengths downstream of the wing trailing edge. The wake rake probes had a spacing of $3 \mathrm{~mm}$ at the center, with decreasing density up to a separation of $24 \mathrm{~mm}$ at the outer edges. The full span of the rake was $2.1 D_{\mathrm{P}}$, which was insufficient to capture the propeller slipstream and wing wake with acceptable resolution in a single traverse along the Y-axis. Accordingly, for each measurement configuration the wake rake was traversed along the wing span twice, centering the rake once around the propeller slipstream and once around the wing wake. Each traverse covered a spanwise interval of $1.7 D_{\mathrm{P}}$, centered around the vertical position of the propeller axis.

\section{II.C. Test conditions}

The wing was set to an angle of attack which was representative of cruise conditions $\left(C_{L} \approx 0.5\right)$ and had been evaluated in earlier experiments, ${ }^{21} \alpha=2.08^{\circ}$. To simulate high-lift conditions, the maximum possible flap deflection of $\delta=23^{\circ}$ was selected, which was limited by the geometry of the support sting. For the selected deflection angle, the values of flap gap and overlap (defined in Figure 3) were selected to be of 3.9\% and 3.5\% chord length respectively, based on earlier reports. ${ }^{21}$
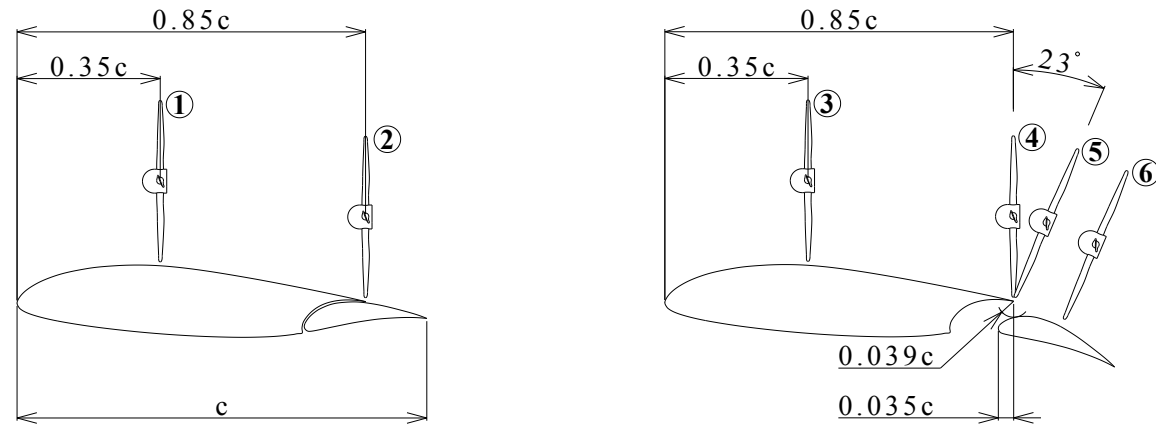

Figure 3. Side view of the six configurations analyzed, separated into flap nested (left) and flap deflected (right) cases. 
Figure 3 shows the selected configurations. For the cruise conditions (flap nested), two axial propeller positions were evaluated: the location of maximum airfoil thickness (Configuration 1, at 35\% chord) and the trailing edge of the main element (Configuration 2, at $85 \%$ chord). These locations were selected based on the observations of earlier studies, ${ }^{9,13,18}$ which indicate that the axial position of maximum wing drag reduction and lift increase are close to the thickest point and the trailing edge respectively. However, the trailing edge of the flap was not considered due to the structural complications that would arise in real applications and the potential reduction of noise shielding effects. The separation between the propeller blade tips and wing surface was chosen as low as possible ${ }^{\mathrm{b}}$, since the drag reduction has been found to be larger for smaller clearances. ${ }^{9}$ The propeller axis was aligned with the freestream direction for simplicity. For the high-lift conditions (flap deflected), two additional propeller positions were evaluated. The first one features a propeller that is located at $85 \%$ chord in cruise conditions (Configuration 2) but is rotated $23^{\circ}$ around the main element's trailing edge when the flap is deflected (Configuration 5). The second one corresponds to the position that the propeller would have attained if it were physically connected to the flap (Configuration 6).

Advance ratios of $0.7,0.8$ and 0.9 were selected for the current experiment, based on isolated propeller data obtained in earlier experiments. ${ }^{22}$ These values correspond to isolated-propeller thrust coefficients of $0.12,0.10$ and 0.06 , respectively. The isolatedpropeller thrust measurements were performed at a wing-chord based Reynolds number of 1.65 million, and thus this Reynolds number was selected for the test campaign, corresponding to a freestream velocity of approximately $41 \mathrm{~m} / \mathrm{s}$. For each setup,

Table 1. Summary of wind tunnel test conditions.

\begin{tabular}{ll} 
Parameter & Test values \\
\hline Reynolds number & $1.65 \cdot 10^{6}$ \\
Wing angle of attack & $2.08^{\circ}$ \\
Flap deflection & $0^{\circ}, 23^{\circ}$ \\
Propeller advance ratio & $0.7,0.8,0.9$ \\
Propeller locations & See Figure 3. \\
\hline
\end{tabular}
a measurement was also taken with the propeller removed and replaced by a dummy hub for reference. A summary of the tested parameters is given in Table 1.

Lift and pressure-drag coefficients were corrected for streamline curvature, wake blockage, and wing blockage $^{23}$ - but not for slipstream blockage, since the propeller disk area represented only $2 \%$ of the crosssectional area of the wind tunnel. Trailing-edge pressures were extrapolated on the suction and pressure sides of the wing, based on the values obtained from the two pressure ports located closest to the trailing edge.

\section{Numerical Model}

To evaluate the performance over-the-wing propellers in a conceptual design phase, a tool that rapidly accounts for the most important interaction effects is necessary. The aim of the tool is to determine the lift and pressure drag of the wing, as well as propeller thrust and torque. Preliminary results of the experimental study confirmed that OTW propellers feature significant two-way interactions. For tractor configurations, the effect of the wing on slipstream displacement or the loading distribution on the propeller disk is often neglected. ${ }^{13}$ However, neglecting this coupling for OTW configurations can lead to inaccurate results, as evidenced in earlier studies. ${ }^{18}$ Therefore, even to model the two-way interaction in the simplest way, an iterative procedure is required. Previous studies have indicated that viscous drag is barely affected by OTW propellers, ${ }^{9}$ and hence an inviscid formulation is selected for simplicity. Furthermore, to reduce computational requirements, it is hypothesized that the unsteady loading on the propeller blades can be represented by means of a steady non-uniform loading distribution on the propeller disk and slipstream. The validity of this hypothesis will be discussed when comparing the results to those of the experimental study.

The numerical model contains three components: the propeller, its slipstream and the wing. A flow diagram of the solver with illustrations of its components is shown in Figure 4. A Panel Method (PM) is used to represent the wing. The velocities induced by the wing are used as input for a modified Blade-Element Model (BEM), from which the time-average distribution of circulation across the propeller disk is obtained. Subsequently, the slipstream is modeled by a time-averaged Vortex Lattice Model (VLM). Once the circulation distribution and position of the slipstream are known, the velocities induced by the slipstream on the wing are computed, and the system is solved iteratively. After each iteration, the wing- and propeller-induced velocities are compared to their value in the previous iteration. Convergence is attained if their root-mean-square difference is within a requested tolerance.

\footnotetext{
${ }^{\mathrm{b}}$ For safety reasons, this was considered to be $6 \mathrm{~mm}$, i.e. $1 \%$ chord.
} 

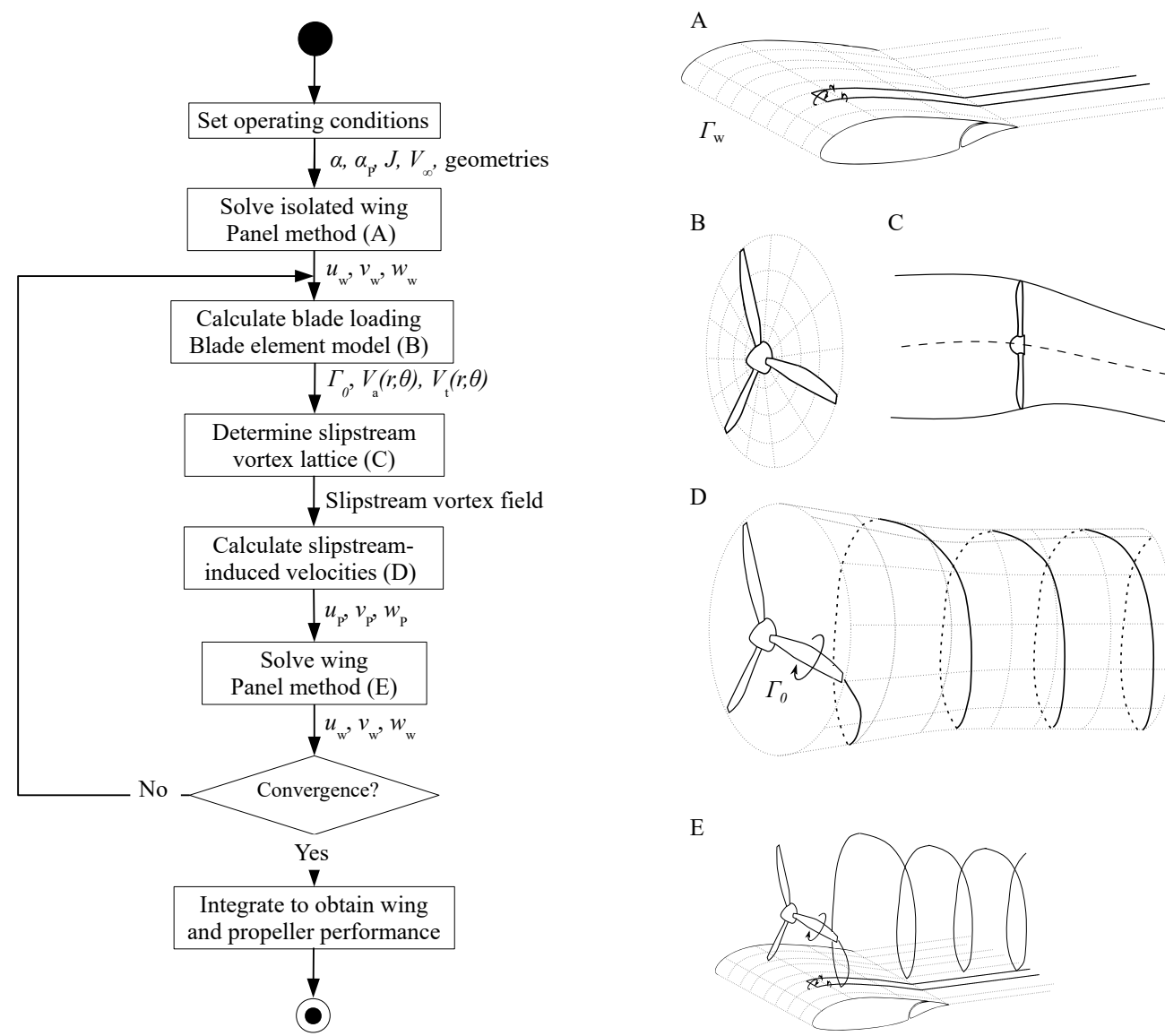

B

$\mathrm{C}$

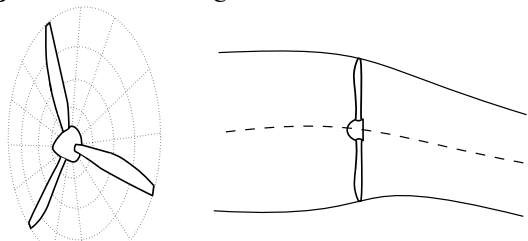

$\mathrm{D}$

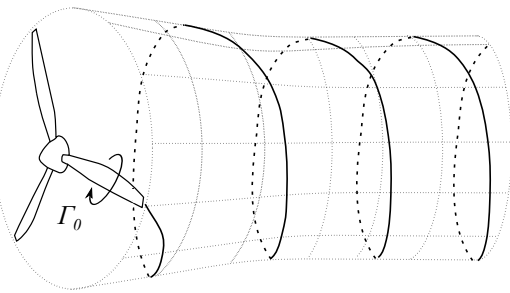

$\mathrm{E}$

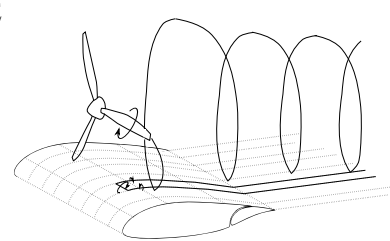

Figure 4. Left: flow diagram of the over-the-wing propeller numerical tool. Right: schematic representation of the elements which comprise the numerical model.

The following sections discuss the PM, BEM, and VLM, as well as the accuracy of the BEM and PM by comparing their results to wind tunnel data. The validation of the complete, coupled model is addressed in Section IV, where it is discussed in tandem with the wind tunnel results.

\section{III.A. Panel method}

To model the wing and nacelle, the existing Fortran-based panel code, FASD (Flow Analysis using Singularity Distributions), ${ }^{24}$ is used. This program divides geometries into rectangular panels, on which a source and sink are placed. The singularity strengths are found by setting appropriate boundary conditions, such as tangent flow at body surfaces. With this, flow properties such as pressure or velocity can be requested at any desired point. Figure 5 illustrates the geometrical setup of the panel method. The velocity field is sampled at the propeller disk and in the slipstream, and used as input for the BEM and slipstream model, respectively. Preliminary experimental results showed that the presence of the nacelle had a noticeable effect on wing performance. However, internal memory limitations in the program prevented the simulation of the complete wind-tunnel setup. Thus, the setup was simplified and represented by means of a single nacelle situated above a high aspect ratio, rectangular wing. Only the central segment of the wing, corresponding to the interval occupied by the propeller, is depicted in Figure 5.

Isolated-wing pressure distributions are shown in Figure 6, as measured in the wind tunnel and simulated in FASD. With the flap retracted, the panel method shows good agreement with the wind tunnel results. However, when it is deflected, the panel method presents unrealistic results in the cove of the main element, even if the kink on the lower side of the airfoil is smoothed (NLF-MOD22A). This is due to the incapability of panel methods to model regions with significant viscous effects or recirculation. Accordingly, in this paper the results for high-lift configurations will be limited to the experimental campaign. 


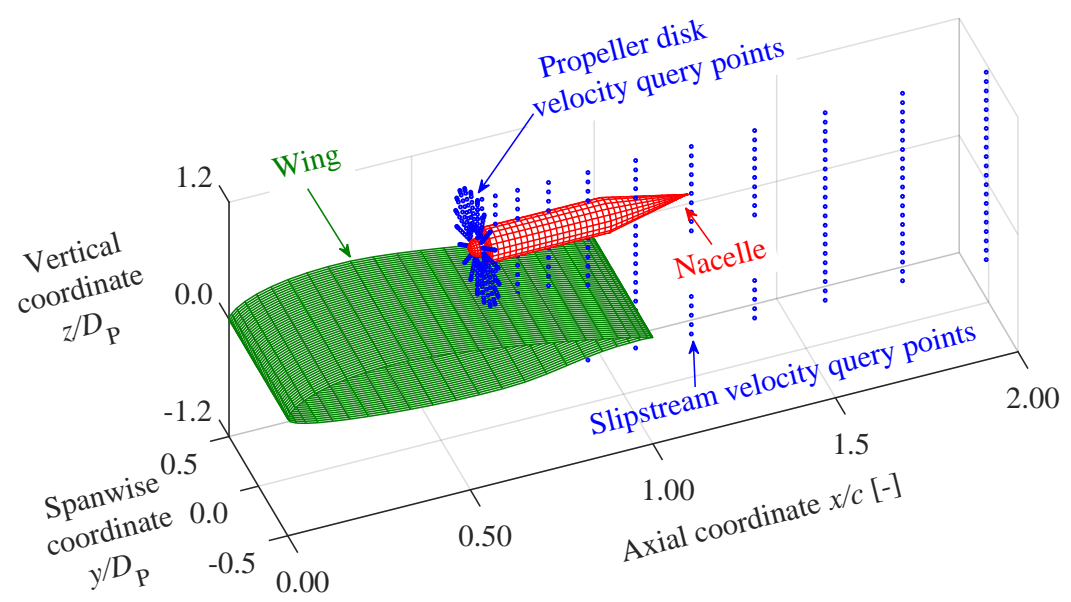

Figure 5. Geometrical representation of the wing segment located under the propeller disk (green) and nacelle (red) modeled in the panel method, indicating the propeller disk and $y=0$ slipstream planes where velocities are requested.

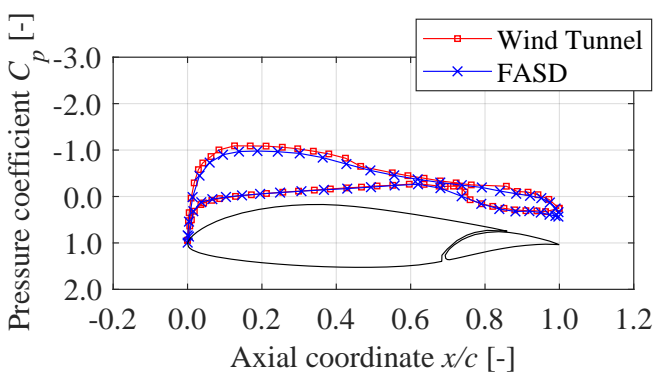

a) Flap nested

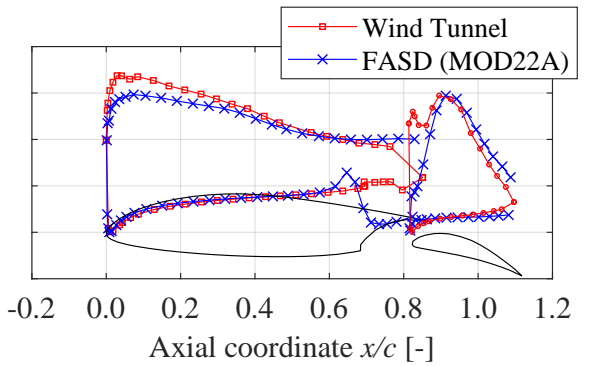

b) Flap deflected

Figure 6. Isolated wing pressure distributions, found during the wind tunnel campaign (red) and by numerical simulation in FASD (blue).

\section{III.B. Blade element model}

Traditional blade element methods divide the propeller disk into radial elements. The disk is assumed to be continuous, and interaction between blades is neglected. In the case of OTW propellers, the inflow is highly non-uniform, and thus the blade element model (BEM) used in this paper is adapted to account for wing-induced velocities. Consequently, the disk is discretized in both radial and azimuthal direction, as depicted in Figure 7a. The loading of each element is then computed independently, taking into account the local inflow conditions as follows. Consider a propeller at angle of attack $\alpha_{\mathrm{p}}$ with rotational speed $\Omega$. A generic blade element, given by its cylindrical coordinates on the propeller disk $\left(r_{0}, \theta_{0}\right)$, is shown in Figure $7 \mathrm{~b}$, together with its simplified velocity diagram. If in the wing coordinate-system the inflow vector is comprised of the freestream velocity plus wing-induced velocities, i.e. $\left(V_{\infty}, 0,0\right)+\left[u_{\mathrm{w}}\left(r_{0}, \theta_{0}\right), v_{\mathrm{w}}\left(r_{0}, \theta_{0}\right), w_{\mathrm{w}}\left(r_{0}, \theta_{0}\right)\right]$, then careful consideration of the velocity components will show that the axial $\left(V_{\mathrm{a}}\right)$ and tangential $\left(V_{\mathrm{t}}\right)$ inflow components at the blade element are:

$$
\begin{gathered}
V_{\mathrm{a}}=\left(\left[V_{\infty}+u_{\mathrm{w}}\left(r_{0}, \theta_{0}\right)\right] \cos \left(\alpha_{\mathrm{p}}\right)-w_{\mathrm{w}}\left(r_{0}, \theta_{0}\right) \sin \left(\alpha_{\mathrm{p}}\right)\right)(1+a), \\
V_{\mathrm{t}}=\left(\Omega r_{0}+\left[V_{\infty}+u_{\mathrm{w}}\left(r_{0}, \theta_{0}\right)\right] \sin \left(\alpha_{\mathrm{p}}\right)-w_{\mathrm{w}}\left(r_{0}, \theta_{0}\right) \cos \left(\alpha_{\mathrm{p}}\right)-v_{\mathrm{w}}\left(r_{0}, \theta_{0}\right) \sin \left(\theta_{0}\right)\right)(1-b),
\end{gathered}
$$

in which $a$ and $b$ are, respectively, the axial and tangential blade induction factors, to be determined iteratively. These velocities can be used to determine the blade section's angle of attack as shown in Figure 7b, after which the lift and drag are determined from the lift and drag polars for the specific blade section. These are created using XFOIL, ${ }^{25}$ based on the NACA 64A416 blade section. ${ }^{26}$ The lift and drag coefficients are then corrected for three-dimensional ${ }^{27}$ and compressibility ${ }^{28}$ (valid up to Mach number 0.9) effects, and hub and tip loss factors ${ }^{29}$ are applied. Subsequently, propeller performance parameters such as thrust, torque, and efficiency can be determined by integrating the forces acting on the blade elements. 

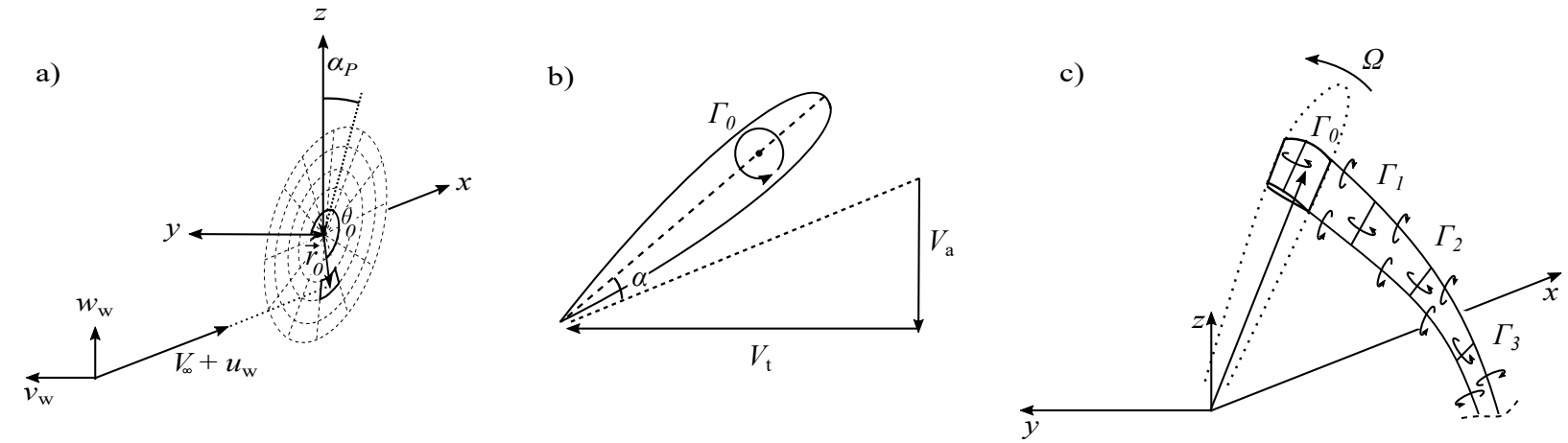

Figure 7. Schematic representation of (a) the discretized propeller disk used in the BEM, (b) the simplified velocity diagram of a generic blade section, and (c) a blade element with its trailing vortices.

Figure 8 presents the radial total-pressure distribution and the thrust coefficient versus advance ratio $J=\frac{V_{\infty}}{n D_{P}}$ curve of the isolated (i.e. uninstalled) propeller, as found in earlier experiments, ${ }^{22}$ and as computed by the BEM. The BEM slightly under-predicts thrust at low advance ratio, and overestimates it at high advance ratio, due to Reynolds number effects which are not modeled.

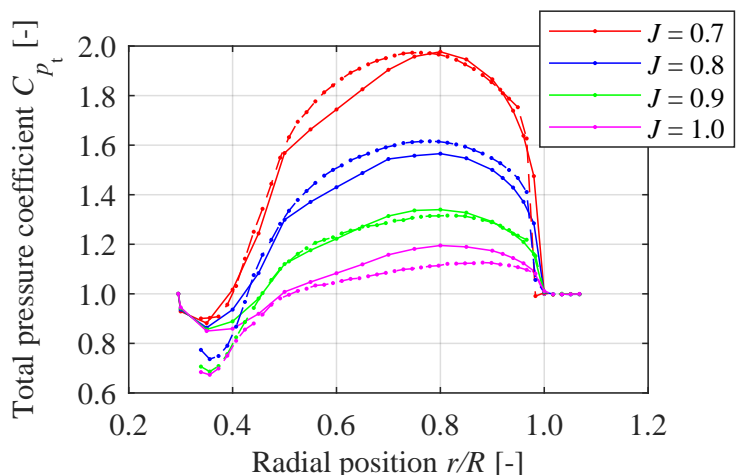

a) Radial blade loading distribution

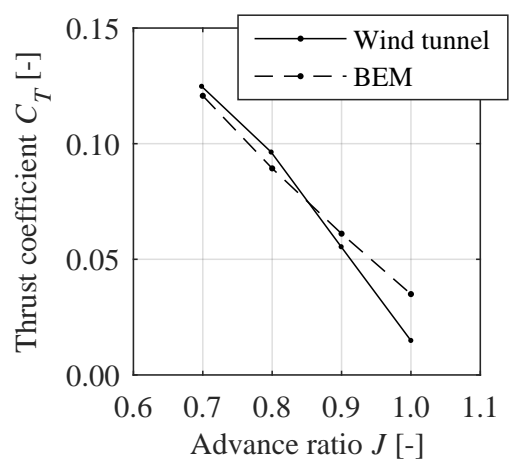

b) Propeller thrust curve

Figure 8. Radial blade loading distribution (a) and thrust coefficient versus advance-ratio curve (b) of the isolated propeller used in this study, comparing results obtained from earlier experiments ${ }^{22}$ with those computed using the BEM.

\section{III.C. Slipstream vortex lattice}

The propeller blade can be represented as a lifting line, whose trailing vortex system is constituted of a radial distribution of horseshoe vortices. In the time-average approach taken here, the individual blades and associated trailing vortex system are replaced by a continuous distribution of trailing vortices, emanating from different locations on the propeller disk. The strength of each trailing vortex is related to the circulation generated on the disk element from which it emanates, as computed by the BEM. Furthermore, since the non-uniform inflow will cause the blade circulation to depend not only on its radial, but also azimuthal position, vortices must be shed in the blade wake to satisfy Kelvin's circulation theorem. This is schematically represented in Figure 7c. Although in the propeller blade reference frame the unsteady loading will lead to periodic, time-dependent vortex shedding, in an inertial reference frame, assuming a continuous propeller disk, this leads to a steady, spatially-periodic distribution of shed vortices.

In the case of an isolated propeller at zero angle of attack, the slipstream only deforms due to contraction. However, for an OTW configuration, the difference in loading between the different regions on the propeller disk can differ notably. ${ }^{19}$ Thus, the effect of non-uniform loading on the deformation of the slipstream must be accounted for. Furthermore, the wing induces velocities which displace the slipstream. Therefore, the resulting slipstream geometry is approximated by a semi-cylindrical tube which is deformed due to contraction, radial and azimuthal variations in loading, and the presence of a wing-induced flow field ${ }^{c}$.

\footnotetext{
${ }^{\mathrm{c}}$ By fixing both the circulation strength and geometrical distribution of the vortices, the flow tangency condition at the outer boundary of the slipstream may be violated. This inconsistency can only by avoided by using a free wake model, which was not chosen in order to limit computational cost.
} 
The geometry of the slipstream geometry is computed as follows. Consider a generic helical vortex line trailing a blade element, as sketched in Figure $7 \mathrm{a}$. The bound vortex on the blade element has a circulation $\Gamma_{0}$, as determined by the BEM. If $\Delta t$ is the duration of each time step in a series of time intervals, then the $j$-th horseshoe vortex in the wake of the propeller blade will have been shed a time $j \Delta t$ earlier. At this time instant, the blade element had angular coordinate $\theta_{0}+j \Omega \Delta t$ and a corresponding circulation $\Gamma_{j}=\Gamma\left(r_{0}, \theta_{0}+j \Omega \Delta t\right)$. Since Kelvin's theorem demands that vorticity must be conserved, when the circulation of the bound vortex changes, the blade element must shed a vortex with equal strength, but opposite direction in its wake, such that $\Gamma_{j}=\Gamma\left(r, \theta_{0}+j \Omega \Delta t\right)-\Gamma_{j-1}$ for $j>0$. The position vector of this shed vortex $j$ (for $j>0$ ) is equal to the position vector of the previous vortex with index $j-1$ and coordinates $\left(x_{j-1}, y_{j-1}, z_{j-1}\right)$, plus:

1. the axial slipstream displacement in the interval $\Delta t, \Delta x=V_{\mathrm{a}} \frac{1+s}{1+a} \Delta t$,

2. the azimuthal displacement of the slipstream helix, $\Delta \theta=\frac{V_{\mathrm{t}}}{r} \Delta t$,

3. the slipstream contraction, $\Delta r$,

4. the wing-induced movement ${ }^{\mathrm{d}},(\Delta x, \Delta z)=\left(u_{\mathrm{w}}\left(x_{j-1}, z_{j-1}\right), w_{\mathrm{w}}\left(x_{j-1}, z_{j-1}\right)\right) \Delta t$,

in which the contraction $\Delta r$ and corresponding acceleration factor, $s$, are determined following the method of Rwigema. ${ }^{29}$ Starting at the bound vortex, the vortex locations and strengths can be determined sequentially until the desired number of axial slipstream elements is reached. After constructing this vortex lattice for all radial and azimuthal positions, the propeller-induced velocities at the wing are computed with Biot-Savart's law. These velocities are then used as input for FASD to capture the influence of the propeller on the wing.

\section{III.D. Limitations and convergence of the numerical model}

Three aspects of the numerical method limit its applicability. Firstly, the BEM compressibility model is limited to propeller blade Mach numbers of 0.9. Secondly, the inviscid nature of the panel method does not allow the modeling of wings where significant viscous effects are present, such as the high-lift configuration depicted in Figure 6b. Thirdly, the use of discrete vortices to represent the slipstream prohibits any meaningful determination of induced velocities inside the slipstream. This prevents convergence for propeller positions near the leading edge, for which the slipstream geometry intersects with the wing surface.

The discretization of the wing, slipstream, and propeller disk also has an important effect on the results. Convergence studies showed that the wing lift coefficient had converged within $0.1 \%$ for 10 radial blade and slipstream elements, 20 azimuthal blade and slipstream elements, 200 axial slipstream elements, and a modeling of the slipstream up to 2.5 chord-lengths behind the propeller disk. Due to Fortran workspace limitations, the number of chord- and spanwise wing panels were restricted to 50 and 125 respectively, with a wing aspect ratio of 6.7 . For these program settings, the wing lift coefficient had converged to within $1 \%$. The numerical tool typically requires three to four iterations, and finishes a complete simulation in approximately ten minutes using a single core on a personal laptop.

\section{Results}

In this section, the wing and wake pressure distributions obtained from the experimental campaign are discussed and compared to the results of the numerical model. Furthermore, the pressure distributions are integrated to assess the effect of the OTW propeller on wing lift and pressure drag. Since the objectives for the cruise and climb conditions (high lift-to-drag ratio and high lift, respectively) are different, they are discussed separately in Sections IV.A and IV.B. Finally, in Section IV.C, the numerical method is applied to analyze the sensitivity of wing lift, pressure drag, and propeller efficiency to top-level design parameter such as the diameter and axial position of the propeller.

\section{IV.A. Performance in cruise-lift conditions}

The following paragraphs present the results for moderate lift coefficient values $\left(C_{L} \approx 0.5\right)$, which are representative of the cruise phase. In these conditions, the flap is nested, and the performance of the wing should be optimized in terms of lift-to-drag ratio.

\footnotetext{
${ }^{d}$ The velocities induced by the wing in spanwise direction were found to be small, and were therefore neglected.
} 


\section{IV.A.1. Effect of propeller on wing performance}

To demonstrate the effect of the propeller on the pressure distribution over the wing surface, Figure 9 presents the pressure coefficient difference between the propeller-on and propeller-off measurements, i.e.

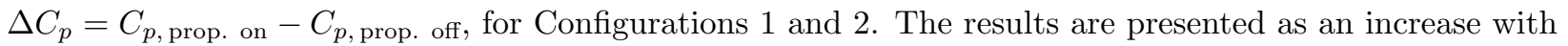
respect to propeller-off measurements because this study focuses on the effect of the propeller, and not the installation effects of a nacelle and support structure of arbitrary geometry. In Figure 9 the flow goes from left to right, and the spanwise coordinate $y / D_{\mathrm{P}}=0$ corresponds to the location of the propeller axis.

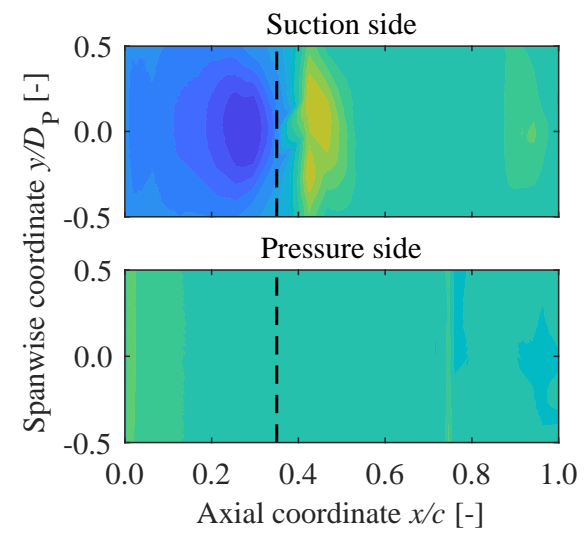

a) Configuration 1 (wind tunnel)
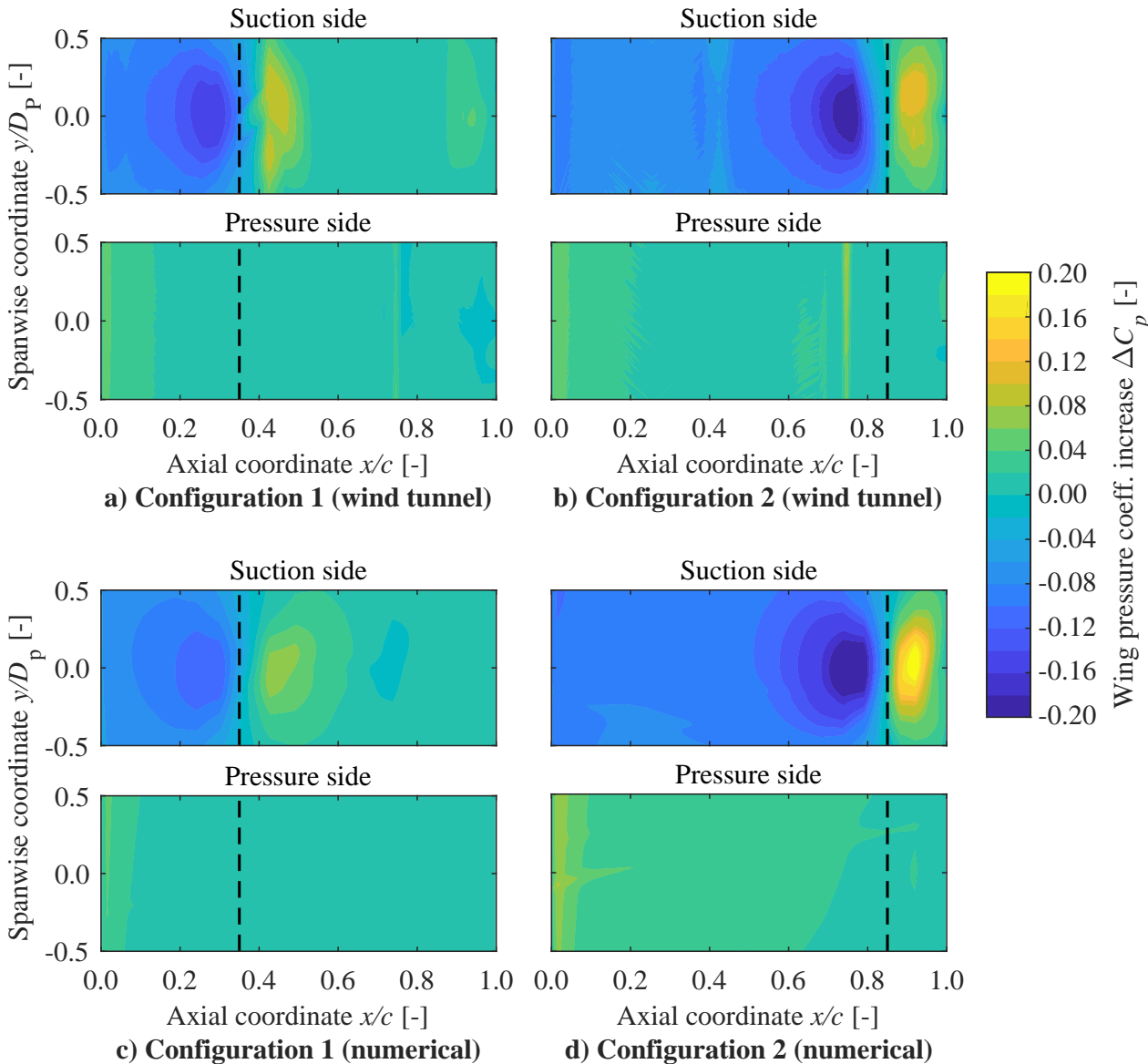

b) Configuration 2 (wind tunnel)
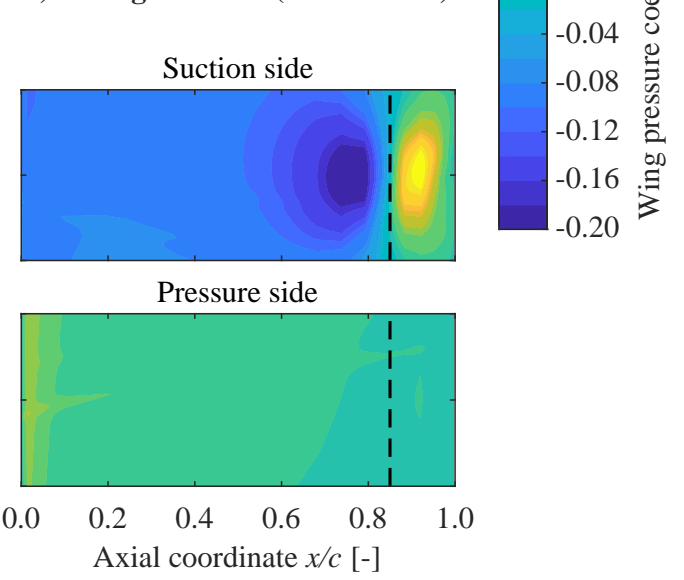

d) Configuration 2 (numerical)

Figure 9. Wing pressure distributions with the flap nested at advance ratio $J=0.7$. Dashed lines indicate the projection of the propeller disk onto the wing surface.

Figure 9 shows that, on the suction side, pressure was reduced in front of the propeller and increased behind it for both configurations. This is attributed to the acceleration of the flow ahead of the propeller, while behind the propeller disk a region of flow diverging from the wing surface is created by the contraction of the slipstream. Results at advance ratios $J=0.8$ and $J=0.9$ (which are not presented here) confirmed that the magnitude of the pressure differences diminished with increasing advance ratio. More pronounced pressure effects of the propeller on the wing are observed for Configuration 2 than for Configuration 1 . This is a consequence of the higher effective advance ratio in Configuration 1 , since the wing-induced velocities were higher at $35 \%$ chord than at the main element's trailing edge (see Figure $6^{\mathrm{e}}$ ). In both cases, the pressure distributions were nearly symmetric with respect to $y / D_{\mathrm{P}}=0$. However, in the experimental results of Configuration 1 (Figures $9 \mathrm{a}$ and $9 \mathrm{~b}$ ), a secondary pressure increase can be seen on the suction side at $x_{p} / c=0.9$. This effect was also present in the numerical simulations, though less pronounced, and is caused by a variation in slipstream-vortex strength near the wing due to the non-uniform propeller loading. On the pressure side, meanwhile, the only clearly-defined pattern is a minor increase in pressure coefficient near the flap slot, due to the increased pressure behind the propeller disk which is propagated through the slot. This effect is absent in the numerical model, which did not include the slot.

eThe isolated-wing pressure distributions give an indication of the local velocity magnitude. For steady, incompressible, inviscid flow the local flow velocity can be expressed as $V=V_{\infty} \sqrt{1-C_{p}}$. 
Comparing Figures $9 \mathrm{a}$ and $9 \mathrm{~b}$ to $9 \mathrm{c}$ and $9 \mathrm{~d}$ shows that the effect of the propeller on the wing was captured correctly by the numerical model. However, the simplification of steady non-uniform loading caused the numerical tool to underestimate and overestimate propeller-induced pressure changes in Configuration 1 and 2, respectively. Furthermore, in Configuration 1 the region of increased pressure directly behind the propeller was predicted too far aft.

The findings of Johnson and White, ${ }^{9}$ who stated that an OTW propeller had no effect on viscous drag, was further assessed by analyzing the IR images. These showed that, for the aft-mounted position (Configuration 2 ), the boundary-layer transition location was not noticeably affected by the propeller. For Configuration 1 , on the other hand, local changes in transition location were observed. These observations are shown in Figure 10. In the propeller-off case of Configuration 1 (Figure 10a), the transition location moved aft due to the favorable pressure gradient generated by the nacelle. However, with the propeller operating ahead of the transition line (Figure 10), the transition location moved forward. There are two reasons for this. Firstly, an adverse pressure gradient is generated in the vicinity of the propeller disk, as visible in Figure 9. Secondly, the interaction between the tip vortices and the boundary layer introduces instabilities in the flow. ${ }^{15}$ Additional studies are required to quantify the relative impact of these effects, but in any case the IR images indicate that the propeller only has an impact on friction drag if it is installed close to or ahead of the transition location.

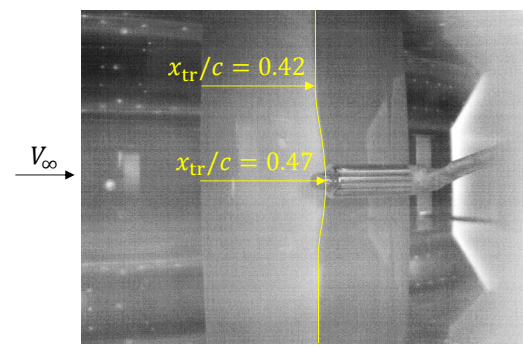

a) Configuration 1, Prop off

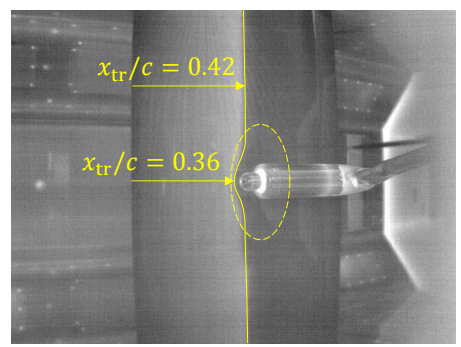

b) Configuration 1, $J=0.7$

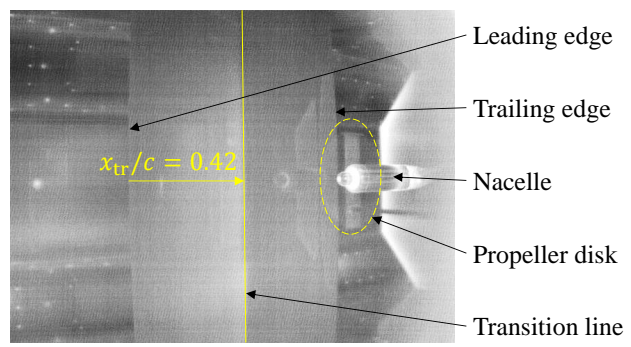

c) Configuration 2, $J=0.7$

Figure 10. Infrared images showing the effect of the propeller on boundary layer transition.

By integrating the pressure distributions, wing lift and pressure drag were obtained. Note that for the computation of these coefficients the reference area considered spans one propeller diameter, that is, forces are evaluated over an area of $S_{\text {ref }}=c \cdot D_{\mathrm{P}}$, and not over the entire wing. For reference, Table 2 presents the isolated wing and propeller-off lift and drag coefficients. Propeller-off pressure measurements differed appreciably from those in Figure 6 due to the blockage effect of the nacelle and support sting, and showed that upstream of the nacelle the static pressure was increased, whereas it decreased directly beneath the nacelle. This generally led to additional pressure drag in all configurations, given that the nacelle was situated above the rearwards-facing part of the wing surface. For nacelle positions near the trailing edge (Configurations 2 and 4-6), most of the wing was ahead of the nacelle, leading to increased pressures and, accordingly, decreased lift. In the numerical simulations, these effects were also observed, but less pronounced, due to the absence of the support sting. Furthermore, Table 2 shows that the numerical simulation overestimates both isolated-wing lift and pressure drag, since it omits of the decambering effect of the

Table 2. Propeller-off and isolated wing lift and drag coefficients for cruise configurations.

\begin{tabular}{ccccc} 
& \multicolumn{2}{c}{ Experimental } & \multicolumn{2}{c}{ Numerical } \\
Configuration & $C_{L}$ & $C_{D_{p}}$ & $C_{L}$ & $C_{D_{p}}$ \\
\hline Isolated wing & 0.47 & 0.006 & 0.50 & 0.007 \\
1 (propeller off) & 0.47 & 0.013 & 0.51 & 0.011 \\
2 (propeller off) & 0.44 & 0.008 & 0.50 & 0.008 \\
\hline
\end{tabular}
boundary layer.

As discussed earlier, the primary interest of this study lies in the effect of the propeller, and not of the installation elements. Accordingly, Figure 11 shows the lift and drag coefficients as a difference between propeller-off and propeller-on conditions. Evidently, wing lift increased with decreasing advance ratio (increased thrust). This can be attributed to the region of low pressure generated in front of the propeller, which became stronger with increasing propeller thrust. In Configuration 1 the wind tunnel measurements at $J=0.9$ present a lower lift coefficient than with the propeller off, due to local windmilling of the blades close to the wing surface. This was reflected in wing pressure distributions, which in these cases presented increased pressure ahead of the propeller disk, contrary to the behavior observed in Figure 9. 


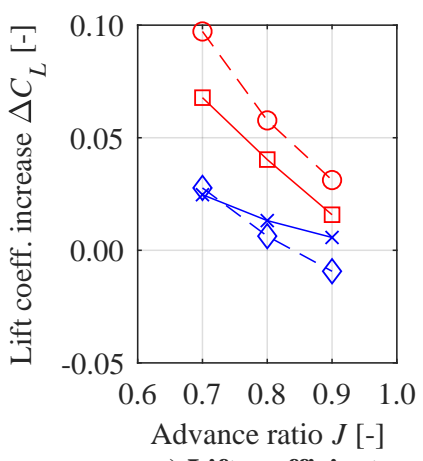

a) Lift coefficient

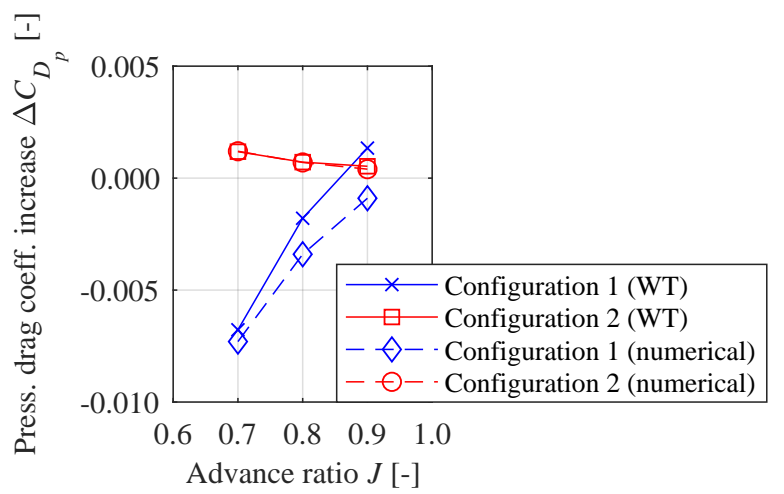

b) Pressure drag coefficient

Figure 11. Wing lift and pressure drag increase with respect to propeller-off conditions as a function of advance ratio.

Regarding the pressure drag of the wing, Figure 11 shows that it decreased with decreasing advance ratio for Configuration 1. This is due to increased suction ahead of the thickest point of the wing. In Configuration 2 , it appears the pressure variations upstream and downstream of the thickest point scaled equally with advance ratio, leading to an approximately constant pressure drag.

The numerical tool presents good qualitative agreement in both configurations. However, it overestimates the lift in Configuration 2, as a consequence of overestimating the pressure differences on the wing surface discussed in Figure 9. This is contrary to the method of Cooper et al., ${ }^{18}$ which heavily underestimates the effect of the propeller on wing performance. Additionally, in Configuration 1, the differences in lift and pressure drag between the experimental and numerical results decrease with increasing advance ratio. This is caused by the overestimation of blade loading by the BEM at high advance ratios (see Figure 8).

\section{IV.A.2. Effect of wing on propeller performance}

The total-pressure distributions in the propeller disk plane and wake plane, obtained from the numerical model and experimental setup respectively, are shown in Figure 12. By comparing Figures 12a and 12c to $12 \mathrm{~b}$ and $12 \mathrm{~d}$, it can be observed how the highly-loaded region in the wake plane has turned in clockwise direction due to the propeller-induced swirl, increasing in magnitude and concentrating over a smaller region due to contraction. In the wake plane (Figures $12 \mathrm{~b}$ and $12 \mathrm{~d}$ ), the decreased total pressure in the wake of the support sting, nacelle and wing can be clearly distinguished. Furthermore, the propeller slipstream is deformed and displaced in vertical direction due to the downwash of the wing.
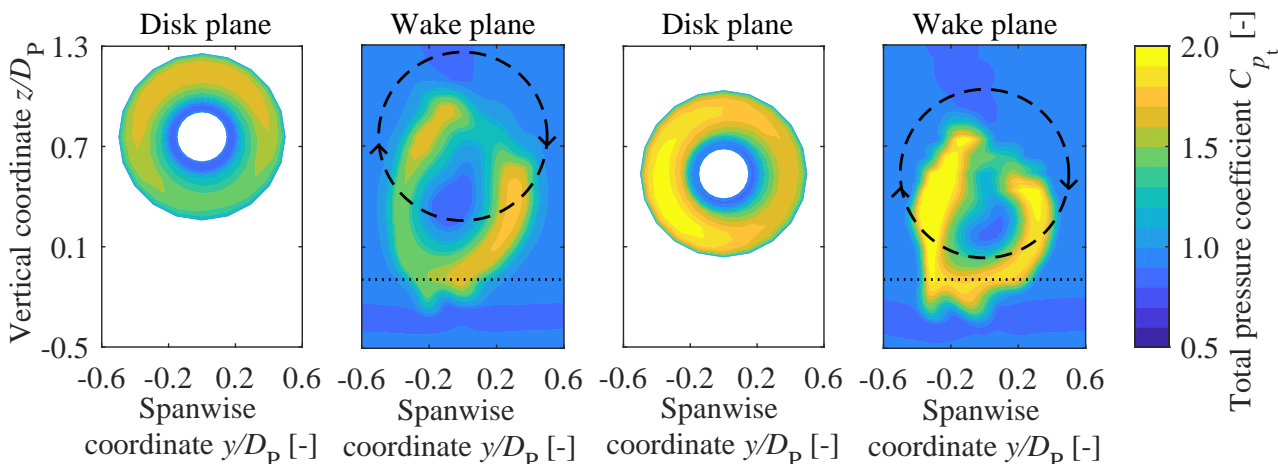

a) Configuration 1 b) Configuration 1 c) Configuration 2 d) Configuration 2

Figure 12. Disk (numerical) and wake plane (experimental) total pressures at advance ratio $J=0.7$. Dashed and dotted lines in the wake plane indicate projections of propeller disk and wing trailing edge, respectively.

Two important effects are observed in Figure 12. Firstly, less thrust was generated than in the isolatedpropeller case in both cruise configurations, due to increased velocities above the wing. This was confirmed by comparing the total pressure distributions in Figures 12a and 12c to the isolated propeller values in Figure 8. The thrust reduction was more pronounced in Configuration 1, since on the forward part of the airfoil the velocity increase generated by the wing was higher, and thus the effective advance ratio of the 
propeller was increased more than in Configuration 2. Secondly, the non-uniform inflow conditions led to azimuthal loading variations in both configurations. In Configuration 1, the strong vertical velocity gradient above the wing was the main source of non-uniform disk loading. This led to a low disk loading near the wing, where inflow velocities were highest. In Configuration 2, however, the wing-induced velocities had a negative $z$-component at the location of the propeller disk, generating the highest loads on the upward-going blade, which experiences a higher angle of attack. From this it can be concluded that, while the effect of the propeller on the wing pressure distribution is symmetric with respect to $y / D_{\mathrm{P}}=0$, the effect of the wing on propeller loading is symmetric in Configuration 1, but asymmetric in Configuration 2.

\section{IV.B. Performance in high-lift conditions}

In this section, the results are presented for high-lift conditions. These conditions are representative of the climb segment, when the flap is deflected $\left(C_{L} \approx 1.6\right)$ and wing lift should be maximized. Although the numerical model does not provide accurate results in case of flap deflection (see Section III), the experimental results are briefly discussed to demonstrate the potential of OTW propellers, and to establish the need for improved methods capable of evaluating OTW systems in high-lift conditions.

\section{IV.B.1. Effect of propeller on wing performance}

The pressure-coefficient distributions on the wing, $\Delta C_{p}$, are presented in Figure 13 for high-lift Configurations 3 to 6 at advance ratio $J=0.7$. Again, wing pressures were decreased and increased in front of and behind the propeller respectively, except in Configuration 3. In this configuration, the effective advance ratio was exceptionally high and, accordingly, propeller effects on the wing were weak. When the propeller was inclined at $23^{\circ}$ (Configuration 5), the suction on the main element was decreased, while the pressure on the flap was increased. The pressure variations became even more prominent in Configuration 6 , due to an improved alignment between the propeller axis and the local flow direction and reduced distance between the flap surface and the propeller.

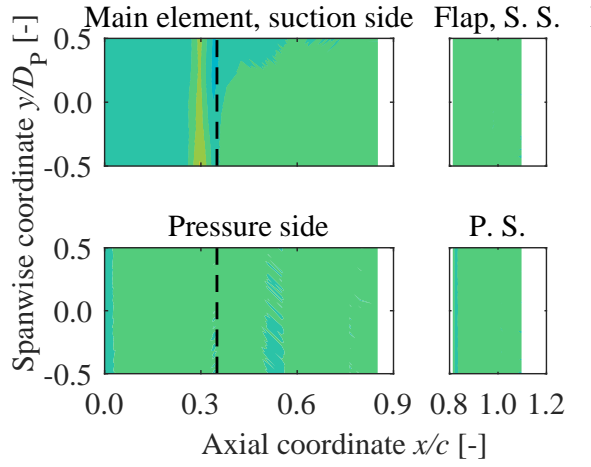

a) Configuration 3
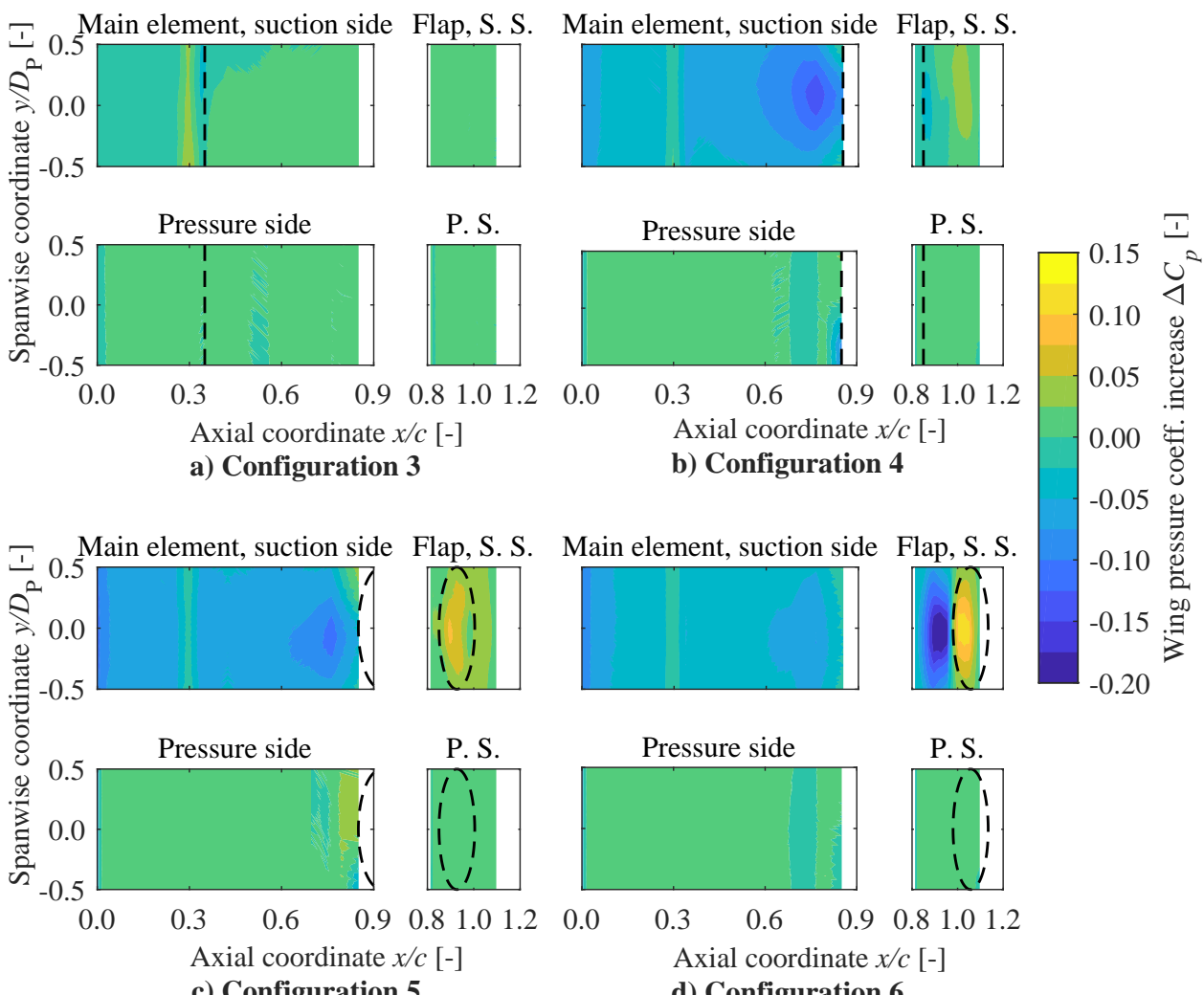

b) Configuration 4

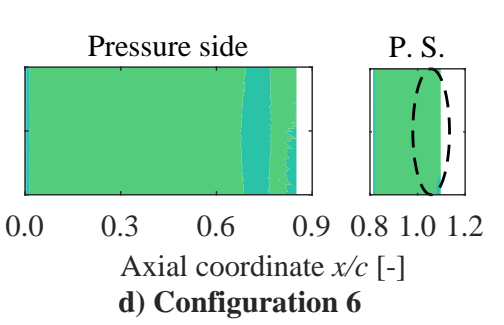

Figure 13. Pressure distributions on the wing with the flap deflected, obtained from the wind-tunnel experiments at $J=0.7$. Dashed lines indicate projection of propeller disk onto the wing surface. Note that the contour levels differ from those in Figure 9. 
Strips of increased pressure can be observed in Figure 13 on the suction side at $x / c=0.3$ for all configurations. IR images revealed that this location corresponded to the chordwise location of boundary-layer transition. Since the transition location was upstream of the propeller for all high-lift configurations, no appreciable changes in transition location were observed. However, a pressure tap was located exactly beneath the transition location, causing a slight movement to show up very prominently.

Analogously to the previous section, Table 3 contains the isolated wing and propeller-off lift and drag coefficients, for reference. Figure 14 shows the lift and drag coefficients as a difference between propeller-on and propeller-off measurements. Figure 14a indicates an increase in lift with decreasing advance ratio, comparable to the effect seen for cruise configurations. From Figure 14 it is evident that at high advance ratios, the propeller was operating in windmilling conditions close to the wing surface, leading to decreased lift and increased pressure drag. This effect was confirmed with the wake-plane pressure distributions (see Section IV.B.2), and was more pronounced in Configuration 3 due to the large inflow velocities perceived by the propeller towards the leading edge of the wing. Again, as the advance ratio decreased, the pressure drag did as well, due to the increased suction

Table 3. Propeller-off and isolated wing lift and drag coefficients for high-lift configurations.

\section{Experimental}

\begin{tabular}{ccc} 
Configuration & $C_{L}$ & $C_{D_{p}}$ \\
\hline Isolated wing & 1.63 & 0.020 \\
3 (propeller off) & 1.64 & 0.032 \\
4 (propeller off) & 1.61 & 0.026 \\
5 (propeller off) & 1.60 & 0.025 \\
6 (propeller off) & 1.60 & 0.023 \\
\hline
\end{tabular}
in front of the wing location with maximum thickness.

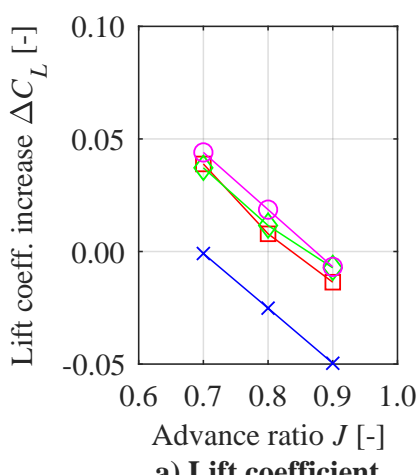

a) Lift coefficient

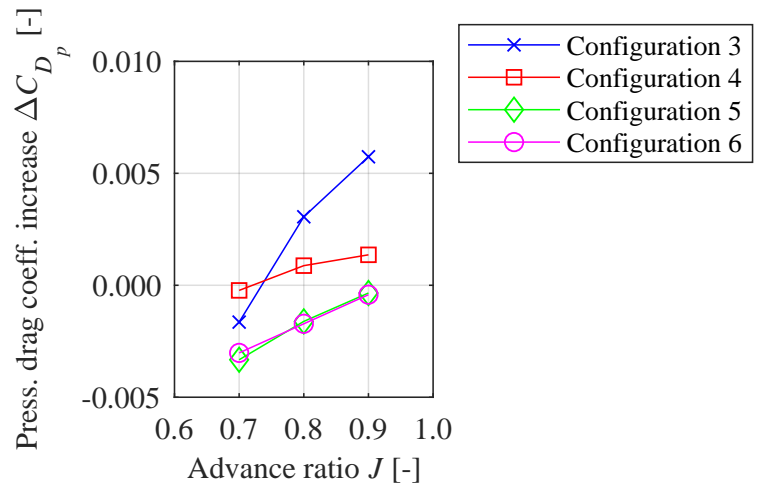

b) Pressure drag coefficient

Figure 14. Wing lift and pressure drag increase with respect to propeller-off conditions in high-lift configurations, as a function of advance ratio.

\section{IV.B.2. Effect of wing on propeller performance}

Results of the total pressure measurements in the wake plane for the climb configurations are shown in Figure 15 at $J=0.7$. Additional wake-plane results showed that, in for example Configuration $3, J=0.8$, the total pressure coefficients in the slipstream were lower than in the freestream, indicating that the propeller was extracting energy from the flow over the complete disk. This implies that in Configuration 3 the propeller was windmilling for advance ratios above 0.8, even though the isolated propeller generated thrust up till $J=1$ (see Figure 8). This explains the lift decrease observed in Figure 14. Since flow velocities above the wing decrease as the distance to the wing surface increases, in some cases only the bottom fraction of the propeller was windmilling, while the top part, which had a lower effective advance ratio, was generating thrust, as reflected in Figure 15a. For the same reason, when comparing Figures 12c, 15a and 15b, it can be seen that the thrust was reduced more in Configuration 3 than in Configurations 1 and 4, since the flow velocities above the wing were higher with the flap deflected and at $35 \%$ instead of $85 \%$ chord-length.

In Figure 15c (Configuration 4), the up-going blade presents higher loading than the down-going blade. This is due to the downward-oriented wing-induced velocities, which follow the local inclination of the airfoil surface. If, on the other hand, the propeller is deflected $23^{\circ}$ (Configurations 5 and 6 ), the angle of attack perceived by the blades is highest on the down-going side. In both Configurations 5 and 6 , the slipstream presents a large vertical displacement due to the inclination of the propeller, and penetrates the wing wake. 
The total pressure values are higher in Configuration 6 than in Configuration 5, since the propeller is ingesting lower-velocity flow. The fact that Configurations 5 and 6 present higher momentum in the slipstream when compared to Configuration 4 indicates that deflecting the propeller together with the flap can lead to increased propeller performance in high-lift conditions.

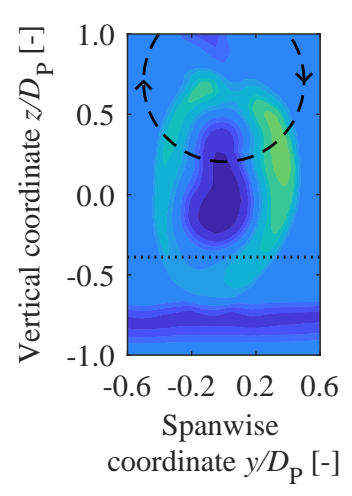

a) Configuration 3

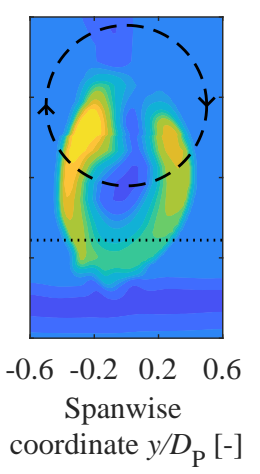

b) Configuration 4

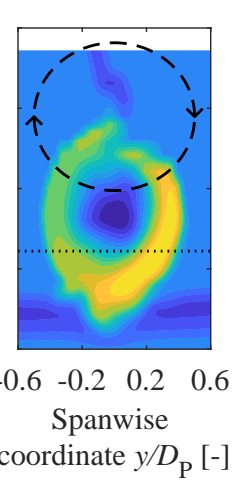

c) Configuration 5

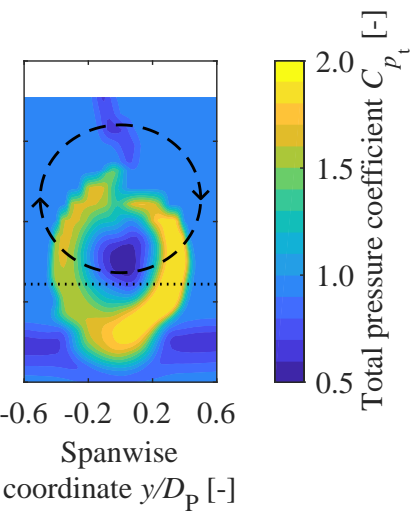

d) Configuration 6

Figure 15. Total-pressure distributions in the wake plane, obtained from the wind-tunnel experiment at $J=0.7$. Dashed and dotted lines indicate projections of propeller disk and wing trailing edge, respectively, onto the measurement plane.

\section{IV.C. Sensitivity analysis: effect of propeller axial location and diameter}

This section demonstrates the applicability of the tool by evaluating the impact of the axial position and size of the propeller. Since Section IV.A.2 has shown that the effective advance ratio - and thus, thrust - varies strongly depending on the configuration, a comparison was performed at constant thrust instead of constant advance ratio. Furthermore, for engineering applications, it is more beneficial to compare wing drag and propeller efficiency at constant lift, instead of constant angle of attack.

Figure 16 presents the lift coefficient, pressure drag coefficient, and propeller efficiency versus propeller axial position for four different thrust coefficient $\left(T_{C}^{*}=\frac{T}{\rho_{\infty} V_{\infty}^{2} S_{\text {ref }}}\right)$ values. The lift and pressure drag coefficients are presented as an increase with respect to propeller-off conditions, while the propeller efficiency is expressed as an increase relative to the isolated propeller efficiency $\eta_{i s o}$. The drag coefficient and propeller efficiency changes are presented at a constant lift, $C_{L}=0.5$. Note that the analysis is limited to axial positions aft of $x_{\mathrm{P}} / c=0.2$, since ahead of this location, the slipstream geometry intersects with the wing surface if the same tip clearance is maintained.

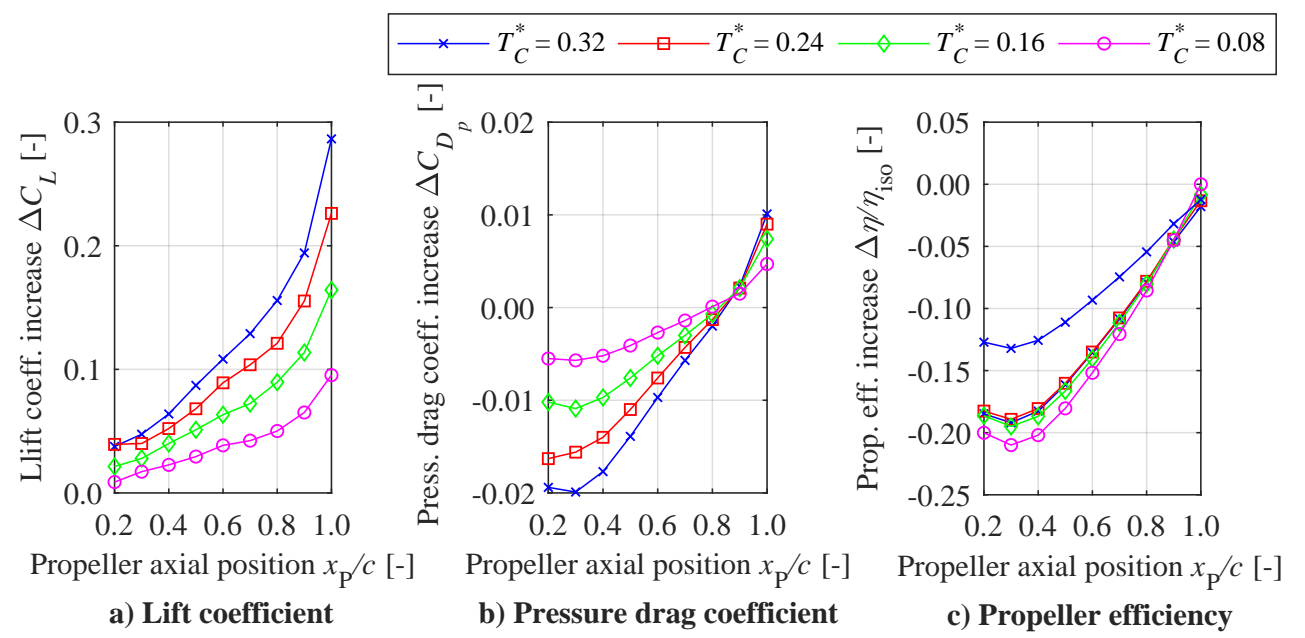

Figure 16. Effect of the axial position of the propeller on the (a) lift increase, (b) pressure drag increase, and (c) propulsive efficiency increase, with respect to propeller-off conditions. The three parameters are evaluated at four constant thrust settings, and (b) and (c) at constant lift $\left(C_{L}=0.5\right)$. 
Regarding the axial position of the propeller, several important trends can be distinguished. Firstly, lift is increased further as the propeller moves aft, due to a larger wing area experiencing the increased suction ahead of the propeller, as explained in Section IV.A.1. At a high thrust setting $\left(T_{C}^{*}=0.32\right)$, the lift coefficient is increased by up to $\Delta C_{L} \approx 0.3$. The effect on pressure drag, on the other hand, is strongly dependent on the axial position of the propeller. At all thrust settings, pressure drag decreases for propeller positions forward of $x_{\mathrm{P}} / c=0.8$, while increasing aft of this position. These trends agree with both the results of the experimental campaign and the findings of Cooper et al. ${ }^{18}$ but not entirely with those of Veldhuis, ${ }^{13}$ who found a maximum lift increase near the mid-chord. The $T_{C}^{*}=0.32$ curve attains a minimum value of $\Delta C_{D_{p}}=-0.02$ near the point of maximum thickness, $x_{\mathrm{P}} / c=0.3$, as expected from earlier studies. ${ }^{9,13,18}$ Surprisingly, at $x_{\mathrm{P}} / c \approx 0.9$ the pressure drag coefficient increase is nearly independent of thrust. This indicates that, at this location, the overall pressure changes on the forward-facing and backward-facing surfaces of the airfoil scale equally with thrust. Finally, the propeller efficiency is lowest near the locations of lowest wing drag, since here the flow velocities above the wing are highest. At $x_{\mathrm{P}}=0.3$, propeller efficiency is decreased by $21 \%$ at $T_{C}^{*}=0.32$. As the propeller moves toward the trailing edge, wing-induced velocities decrease, and the efficiency penalty is reduced to approximately $1 \%$ at the trailing edge. This illustrates that the optimal propeller position in an over-the-wing configuration is most importantly a trade-off of wing lift and propeller efficiency versus wing drag. A propeller position near the wing trailing edge is recommended, since there the lift increase is highest, while drag and propulsive efficiency penalties are limited.

For distributed-propulsion applications, it is important to analyze the effect of propeller diameter, which can differ considerably depending on the number of propulsors selected. This parameter is difficult to vary experimentally, whereas it is easily changed in the numerical model. To this end, Figure 17 presents $\Delta C_{L}$, $\Delta C_{D_{p}}$, and $\Delta \eta / \eta_{i s o}$ versus the propeller diameter, expressed as a fraction of the wing chord. The diameter of the nacelle was scaled linearly with propeller diameter. Based on the results of Figure 16, an axial position of $x_{\mathrm{p}} / c=0.95$ was selected. The lower bound of the diameter interval was limited by the tip Mach number, which increases considerably for smaller propellers if they have to produce large $T_{C}^{*}$ values.

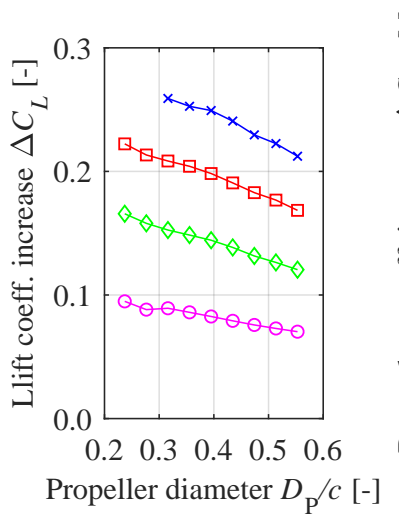

a) Lift coefficient

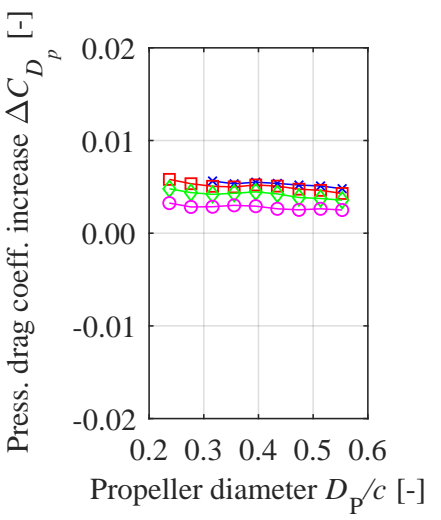

b) Pressure drag coefficient

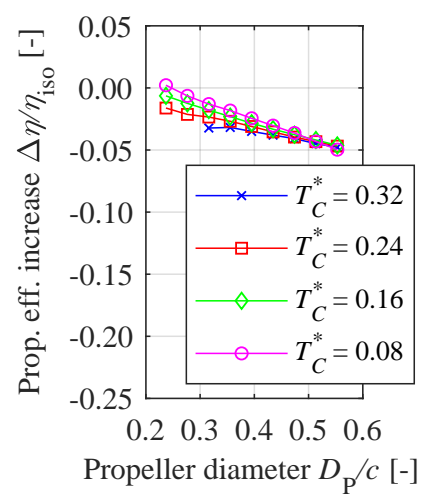

c) Propeller efficiency

Figure 17. Effect of propeller diameter on (a) the wing lift increase, (b) wing pressure drag increase, and (c) propulsive efficiency increase, with respect to propeller-off conditions. The three parameters are evaluated at $x_{\mathrm{P}} / c=0.95$ for four constant thrust settings, and (b) and (c) at constant lift $\left(C_{L}=0.5\right)$.

Figure 17 shows that, within the interval studied, the propeller diameter has less effect on the three parameters than the axial position of the propeller. Nonetheless, it can be seen that wing lift and propeller efficiency are increased as the propeller diameter is reduced, while pressure drag is practically unchanged. This is beneficial from the perspective of distributed-propulsion systems if the number of propellers is increased while maintaining the same total thrust, disk area, and advance ratio. In this case, the isolated propeller efficiency remains constant, and the installed propeller efficiency is increased as its diameter is reduced. Note that the previous conclusion is valid specifically for the axial position considered in Figure 17, $x_{\mathrm{P}} / c=0.95$. Close to the trailing edge, the velocity gradient at the propeller disk is relatively small, and the inflow angle has a positive effect on propeller efficiency ${ }^{30}$ If the propeller were positioned at, for example, $x_{\mathrm{P}} / c=0.35$, the propulsive efficiency would likely decrease as the propeller diameter is reduced, due to the higher inflow velocities closer to the wing. The propeller diameter is also expected to have a larger impact on pressure drag if the propeller is positioned at a different axial location, where the drag changes are higher. 


\section{Conclusions}

The aerodynamic interaction effects and performance of OTW propeller systems have been evaluated in a two-step approach. Experimental results confirm that the propeller decreases the pressure on the wing surface upstream of the propeller disk, while decreasing the pressure behind it. The opposite happens when the propeller is windmilling close to the wing surface, due to the increased flow velocities above the wing. Results indicate that the propeller design must differ significantly from the design of a tractor propeller in order to allow an efficient propeller operation. Promising results have been obtained for a propeller located at $85 \%$ of the wing chord. Although for this position the pressure drag is increased by approximately 2 counts with respect to an isolated wing when the flap is retracted, wing lift is improved by $8 \%$. In high-lift conditions (flap deflected), wing lift increased by $3 \%$ and the pressure drag is reduced by 3 counts. These values are conservative, since the benefits in terms of wing lift and pressure drag were found to increase with decreasing advance ratio. Moreover, an increase in effective lift can be obtained by deflecting the propeller together with the flap, leading to improved propeller performance when compared to the same propeller without deflection.

A numerical tool has been developed, which provides a good estimation of OTW system performance in cruise conditions, although it underestimates the propeller loading for mid-wing positions. Besides supporting the conclusions drawn during the wind tunnel campaign and giving more insight into the experimental results, the tool allows rapid design-space explorations of over-the-wing propeller configurations. Results indicate that the optimal axial location for an OTW propeller is near the wing trailing edge, where it increases lift the most, with moderate increases and decreases in pressure drag and propulsive efficiency, respectively. At this axial location, wing lift and propeller efficiency increase as the diameter of the propeller is decreased, which is beneficial for distributed-propulsion applications.

Based on the findings of this study, future research on OTW propellers will focus on the aerodynamic performance of such systems in high-lift conditions, as well as on the performance of propellers subjected to non-uniform inflow conditions. Additionally, a the numerical model will be adapted to incorporate boundary-layer effects, and elaborated to include the effect of multiple propellers. The resulting method will be invaluable in the conceptual design phase of radical aircraft concepts featuring over-the-wing distributed propulsion.

\section{Acknowledgements}

This research is part of the EU Horizon 2020 Clean Sky 2 program (CS2-LPA-GAM-2016-2017-01). The authors would like to thank the other partners of LPA WP1.6 and the participants of the propeller research group at TU Delft for their valuable feedback.

\section{References}

\footnotetext{
${ }^{1}$ Sehra, A. K. and Whitlow, W., "Propulsion and power for 21st century aviation," Progress in Aerospace Sciences, Vol. 40, No. 4-5, 2004, pp. 199-235.

${ }^{2}$ Bonet, J.T., Schellenger, H.G., Rawdon, B.K., Elmer, K.R., Wakayama, S.R., Brown, D.L. and Guo, Y., "N+2 Advanced Vehicle Concepts Study and Conceptual Design of Subscale Test Vehicle (STV)," Environmentally Responsible Aviation (ERA) Project, 2011.

${ }^{3}$ Advisory Council for Aviation Research and Innovation in Europe (ACARE), "Realising Europes Vision for Aviation: Strategic Research \& Innovation Agenda, Volume 1," Advisory Council for Aviation Research and Innovation in Europe (ACARE), 2012.

${ }^{4}$ Kim, H. D., "Distributed Propulsion Vehicles," International Congress of the Aeronautical Sciences, Nice, France, September 19-24 2010.

${ }^{5}$ Isikveren, A. T., Pornet, C., Vratny, P. C., and Schmidt, M., "Optimization of Commercial Aircraft Using Battery-Based Voltaic-Joule/Brayton Propulsion," Journal of Aircraft, Vol. 54, No. 1, 2017, pp. 246-261.

${ }^{6}$ Pornet, C. and Isikveren, A. T., "Conceptual design of hybrid-electric transport aircraft," Progress In Aerospace Sciences, Vol. 79, 2015, pp. 114-135.

${ }^{7}$ Felder, J. L., Kim, H. D., and Brown, G. V., "Turboelectric Distributed Propulsion Engine Cycle Analysis for HybridWing-Body Aircraft," 47th AIAA Aerospace Sciences Meeting, Orlando, FL, USA, January 5-8 2009.

${ }^{8}$ Borer, N. K., Derlaga, J. M., Deere, K. A., Carter, M. B., Viken, S. A., Patterson, M. D., Litherland, B. L., and Stoll, A. M., "Comparison of Aero-Propulsive Performance Predictions for Distributed Propulsion Configurations," 55th AIAA Aerospace Sciences Meeting, Grapevine, TX, USA, January 9-13 2017.

${ }^{9}$ Johnson Jr., J. L. and White, E. R., "Exploratory Low-Speed Wind-Tunnel Investigation of Advanced Commuter Configurations Including an Over-the-Wing Propeller," AIAA Aircraft Design, Systems and Technology Meeting, Fort Worth, TX, USA, October 17-19 1983.
} 
${ }^{10}$ Müller, L., Heinze, W., Kožulović, D., Hepperle, M., and Radespiel, R., "Aerodynamic Installation Effects of an Over-the-Wing Propeller on a High-Lift Configuration," Journal of Aircraft, Vol. 51(1), 2014, pp. 249-258.

${ }^{11}$ Beck, S. C., Müller, L., and Langer, S. C., "Numerical assessment of the vibration control effects of porous liners on an over-the-wing propeller configuration," CEAS Aeronautical Journal, Vol. 7, No. 2, 2016, pp. $275-286$.

$1{ }^{12}$ Broadbent, E. G., "Noise shielding for aircraft," Progress In Aerospace Sciences, Vol. 17, 1977, pp. $231-268$.

${ }^{13}$ Veldhuis, L. L. M., Propeller Wing Aerodynamic Interference, Ph.D. thesis, TU Delft, 2005.

${ }^{14}$ Magliozzi, B., Hanson, D. B., and Amiet, R. K., "Propeller and Propfan Noise," NASA Langley Research Center, Aeroacoustics of Flight Vehicles: Theory and Practice, Volume 1: Noise Sources, pp. 1-64, 1991.

${ }^{15}$ Müller, L. and Friedrichs, J., "Unsteady Flow Simulations of an Over-the-Wing Propeller Configuration," 50th AIAA Joint Propulsion Conference, Cleveland, OH, USA, 2014.

${ }^{16}$ Patterson, M. D., Conceptual Design of High-Lift Propeller Systems for Small Electric Aircraft, Ph.D. thesis, Georgia Institute of Technology, 2016.

${ }^{17}$ Alba, C., Elham, A., Veldhuis, L., and German, J. G., "A Surrogate-Based Multi-Disciplinary Design Optimization Framework Exploiting Wing-Propeller Interaction," 18th AIAA/ISSMO Multidisciplinary Analysis and Optimization Conference, Denver, CO, USA, June 5-9 2017.

${ }^{18}$ Cooper, R. K., McCann, W. J., and Chapleo, A. Q., "Over wing propeller aerodynamics," ICAS Proceedings (AIAA), Vol. 18, 1992, pp. 266-273.

${ }^{19}$ Müller, L., Hepperle, M., and Radespiel, R., "The Influence of the Propeller Position on the Aerodynamics of a Channel Wing," 61er Deutscher Luft- und Raumfahrtkongress, Berlin, Germany, September 10-12 2012.

${ }^{20}$ Veldhuis, L. L. M., "Configuration and Propulsion aerodynamics research on the Low Speed Aerodynamics laboratory (in Dutch), Review of project 90-LR-5-S," Internal Report LSW 93-1, Fac. of Aerospace Engineering, Delft University of Technology, 1993.

${ }^{21}$ Boermans, L. M. M. and Rutten, P. B., "Two-dimensional Aerodynamic Characteristics of Airfoil NLF-MOD22 with Fowler Flap," TU Delft Internal Report LSW-95-3, 1995.

${ }^{22}$ Sinnige, T., de Vries, R., Della Corte, B., Avallone, F., Ragni, D., Eitelberg, G., and Veldhuis, L. L. M., "Unsteady Pylon Loading Caused by Propeller-Slipstream Impingement for Tip-Mounted Propellers," Journal of Aircraft, in press.

${ }^{23}$ Garner, H. C., Rogers, E., Acum, W., and Maskell, E., "Subsonic wind tunnel wall corrections," AGARDograph, AG-109, 1966, p. 466.

${ }^{24}$ Box, W. and Hoeijmakers, H., "Toepassing van de FASD panelenmethode voor de predictie van het drukveld opgewekt door een passerende trein," TU Eindhoven, 1994.

${ }^{25}$ Drela, M., "XFOIL 6.9 User Primer," 2001.

${ }^{26}$ Biermann, D. and Hartman, E.P., "Tests of Five Full-Scale Propellers in the Presence of a Radial and a Liquid-Cooled Engine Nacelle, Including Tests of Two Spinners," NACA Report No. 642, 1938.

${ }^{27}$ Snel, H. and Schepers, J. G., "Investigation and modelling of dynamic inflow effects," ECWEC Conference, 1993.

${ }^{28}$ Dorfling, J. and Rokhsaz, K., "Integration of Airfoil Stall and Compressibility Models into a Propeller Blade Element Model," J. Aerosp. Eng., Vol. 29, No. 04016014, 2016, pp. 1-14.

${ }^{29}$ Rwigema, M., "Propeller blade element momentum theory with vortex wake deflection," 27 th International Congress of the Aeronautical Sciences, Nice, France, September 2010.

${ }^{30}$ Veldhuis, L. L. M., Berkel, E. V., Kotsonis, M., and Eitelberg, G., "Non-Uniform Inflow Effects on Propeller Performance," 31st AIAA Applied Aerodynamics Conference, June 24-27 2013. 


\section{This article has been cited by:}

1. Reynard de Vries, Roelof Vos. Aerodynamic Performance Benefits of Over-the-Wing Distributed Propulsion for Hybrid-Electric Transport Aircraft . [Abstract] [PDF] [PDF Plus]

2. Caterina Poggi, Giovanni Bernardini, Massimo Gennaretti. Aeroacoustic analysis of wing-mounted propeller arrays . [Abstract] [PDF] [PDF Plus]

3. Reynard de Vries, Nando van Arnhem, Francesco Avallone, Daniele Ragni, Roelof Vos, Georg Eitelberg, Leo L. M. Veldhuis. 2021. Experimental Investigation of Over-the-Wing Propeller-Boundary-Layer Interaction. AIAA Journal 59:6, 2169-2182. [Abstract] [Full Text] [PDF] [PDF Plus] [Supplementary Material]

4. Sören Bölk, Reynard de Vries, Nando v. Arnhem, Leo L. Veldhuis. Numerical Investigation of Propeller-Flap Interaction in Inclined Over-the-Wing Distributed-Propulsion Systems . [Abstract] [PDF] [PDF Plus]

5. D. Felix Finger, Carsten Braun, Cees Bil. 2020. Comparative Assessment of Parallel-Hybrid-Electric Propulsion Systems for Four Different Aircraft. Journal of Aircraft 57:5, 843-853. [Abstract] [Full Text] [PDF] [PDF Plus]

6. Hugo F. Bento, Reynard de Vries, Leo L. Veldhuis. Aerodynamic Performance and Interaction Effects of Circular and Square Ducted Propellers . [Abstract] [PDF] [PDF Plus]

7. Reynard de Vries, Maurice Hoogreef, Roelof Vos. Aero-Propulsive Efficiency Requirements for Turboelectric Transport Aircraft . [Abstract] [PDF] [PDF Plus]

8. Maurice Hoogreef, Reynard de Vries, Tomas Sinnige, Roelof Vos. Synthesis of Aero-Propulsive Interaction Studies Applied to Conceptual Hybrid-Electric Aircraft Design . [Abstract] [PDF] [PDF Plus]

9. D. Felix Finger, Carsten Braun, Cees Bil. Comparative Assessment of Parallel-Hybrid-Electric Propulsion Systems for Four Different Aircraft . [Abstract] [PDF] [PDF Plus]

10. Francesco Orefice, Pierluigi Della Vecchia, Danilo Ciliberti, Fabrizio Nicolosi. Aircraft Conceptual Design Including Powertrain System Architecture and Distributed Propulsion . [Citation] [PDF] [PDF Plus]

11. Reynard de Vries, Nando van Arnhem, Francesco Avallone, Daniele Ragni, Roelof Vos, Georg Eitelberg, Leo L. Veldhuis. Aerodynamic Interaction Between an Over-the-Wing Propeller and the Wing Boundary-Layer in Adverse Pressure Gradients . [Citation] [PDF] [PDF Plus]

12. Reynard de Vries, Maurice Hoogreef, Roelof Vos. Preliminary Sizing of a Hybrid-Electric Passenger Aircraft Featuring Over-theWing Distributed-Propulsion . [Citation] [PDF] [PDF Plus]

13. Reynard de Vries, Malcom T. Brown, Roelof Vos. A Preliminary Sizing Method for Hybrid-Electric Aircraft Including AeroPropulsive Interaction Effects . [Citation] [PDF] [PDF Plus] 